\title{
Stellar Stream and Halo Structure in the Andromeda Galaxy from a Subaru/Hyper Suprime-Cam Survey*
}

\author{
Yutaka Komiyama $^{1,2}$, Masashi Chiba ${ }^{3}$ (D), Mikito Tanaka ${ }^{4}$, Masayuki Tanaka ${ }^{1}$ (1), Takanobu Kirihara ${ }^{5,17}$, Yohei Miki ${ }^{6,7,18}$, \\ Masao Mori ${ }^{5,6}$, Robert H. Lupton ${ }^{8}$ (1), Puragra Guhathakurta ${ }^{9}$ (i), Jason S. Kalirai ${ }^{10,11}$, Karoline Gilbert ${ }^{10,11^{1}}$ (i), Evan Kirby ${ }^{12}$ (1), \\ Myun Gyoon Lee ${ }^{13}$, In Sung Jang ${ }^{13}$, Sanjib Sharma ${ }^{14}$ (1), and Kohei Hayashi ${ }^{15,16,19}$ (1) \\ ${ }^{1}$ National Astronomical Observatory of Japan, 2-21-1 Osawa, Mitaka, Tokyo 181-8588, Japan; komiyama@subaru.naoj.org \\ ${ }^{2}$ Graduate University for Advanced Studies (SOKENDAI), 2-21-1 Osawa, Mitaka, Tokyo 181-8588, Japan \\ ${ }^{3}$ Astronomical Institute, Tohoku University, Aoba-ku, Sendai 980-8578, Japan \\ ${ }^{4}$ Frontier Research Institute for Interdisciplinary Sciences, Tohoku University, Aoba-ku, Sendai 980-8578, Japan \\ ${ }^{5}$ Faculty of Pure and Applied Physics, University of Tsukuba, Tennodai 1-1-1, Tsukuba, Ibaraki, 305-8577, Japan \\ ${ }^{6}$ Center for Computational Sciences, University of Tsukuba, Tennodai 1-1-1, Tsukuba, Ibaraki, 305-8577, Japan \\ ${ }^{7}$ CREST, JST, Tennodai 1-1-1, Tsukuba, Ibaraki, 305-8577, Japan \\ ${ }^{8}$ Department of Astrophysical Sciences, Princeton University, 4 Ivy Lane, Princeton, NJ 08544, USA \\ ${ }^{9}$ UCO/Lick Observatory and Department of Astronomy and Astrophysics, University of California, 1156 High Street, Santa Cruz, CA 95064, USA \\ ${ }^{10}$ Space Telescope Science Institute, Baltimore, MD 21218, USA \\ ${ }^{11}$ Center for Astrophysical Sciences, Johns Hopkins University, Baltimore, MD 21218, USA \\ 12 California Institute of Technology, 1200 E. California Boulevard, MC 249-17, Pasadena, CA 91125, USA \\ ${ }^{13}$ Department of Physics and Astronomy, Seoul National University, Seoul 151-742, People's Republic of Korea \\ ${ }^{14}$ Sydney Institute for Astronomy, School of Physics, University of Sydney, NSW 2006, Australia \\ ${ }^{15}$ Kavli Institute for Astronomy and Astrohysics, Peking University, Beijing 100871, People's Republic of China \\ ${ }^{16}$ Kavli Institute for the Physics and Mathematics of the Universe (Kavli IPMU, WPI), The University of Tokyo, Chiba 277-8583, Japan \\ Received 2017 June 27; revised 2017 November 9; accepted 2017 December 8; published 2018 January 19
}

\begin{abstract}
We present wide and deep photometry of the northwestern part of the halo of the Andromeda galaxy (M31) using Hyper Suprime-Cam on the Subaru Telescope. The survey covers a $9.2 \mathrm{deg}^{2}$ field in the $g, i$, and NB515 bands and shows a clear red giant branch (RGB) of M31's halo stars and a pronounced red clump (RC) feature. The spatial distribution of RC stars shows a prominent stream feature, the Northwestern (NW) Stream, and a diffuse substructure in the southern part of our survey field. We estimate the distances based on the RC method and obtain $(m-M)=24.63 \pm 0.191$ (random) \pm 0.057 (systematic) and $24.29 \pm 0.211$ (random) \pm 0.057 (systematic) mag for the NW Stream and diffuse substructure, respectively, implying that the NW Stream is located behind M31, whereas the diffuse substructure is located in front of it. We also estimate line-of-sight distances along the NW Stream and find that the southern part of the stream is $\sim 20 \mathrm{kpc}$ closer to us relative to the northern part. The distance to the NW Stream inferred from the isochrone fitting to the color-magnitude diagram favors the RC-based distance, but the tip of the RGB (TRGB)-based distance estimated for NB515-selected RGB stars does not agree with it. The surface number density distribution of RC stars across the NW Stream is found to be approximately Gaussian with an FWHM of $\sim 25 \operatorname{arcmin}(5.7 \mathrm{kpc})$, with a slight skew to the southwest side. That along the NW Stream shows a complicated structure, including variations in number density and a significant gap in the stream.
\end{abstract}

Key words: galaxies: halos - galaxies: individual (M31) - galaxies: structure

\section{Introduction}

Faint stellar halos in disk galaxies like the Milky Way (MW) and Andromeda (M31) serve as fossil records of the formation of such galaxies through hierarchical assembly and past accretion events. In the MW, stars spread over the vast reaches of its halo region are characterized by low metal abundance and high velocity dispersion. The extreme nature of halo stars, compared to stars comprising the disk component, reflects the early dynamical and chemical evolution of the MW, when its appearance differed significantly from what we see today. Extensive analyses of such metal-deficient, old populations have revealed various fundamental properties of the Galactic

\footnotetext{
* Based on data collected at the Subaru Telescope, which is operated by the National Astronomical Observatory of Japan.

17 Current Address: Institute of Management and Information Technologies, Chiba University, 1-33, Yayoi-cho, Inage-ku, Chiba 263-8522, Japan.

18 Current Address: Information Technology Center, The University of Tokyo, 5-1-5 Kashiwanoha, Kashiwa, Chiba 277-8589, Japan.

19 Current Address: National Astronomical Observatory of Japan, 2-21-1 Osawa, Mitaka, Tokyo 181-8588, Japan.
}

halo, e.g., its multiple nature in several aspects, including its spatial structure, velocity distribution, and chemical abundances (e.g., Feltzing \& Chiba 2013); the Galactic halo consists of at least two overlapping components: an inner, flattened halo component having a high $[\alpha / \mathrm{Fe}]$ ratio and an outer halo characterized by a more spherical shape, lower metallicity, and lower $[\alpha / \mathrm{Fe}]$ ratio. This complicated global structure of the Galactic halo together with recent growing observational evidence for an abundance of stellar streams and other substructures (e.g., Belokurov et al. 2006) suggests that the stellar halo has formed, at least in its outer part, largely from an assembly process of many subsystems, such as dwarf galaxies, as opposed to an in situ dissipative collapse in its inner part. Indeed, this formation picture of stellar halos is suggested by recent numerical simulations of galaxy formation based on standard ACDM cosmology (Font et al. 2011). Thus, detailed studies of the halo properties can provide important clues to the understanding of galaxy formation.

M31's halo, the target of this work, is another excellent testbed for understanding galaxy formation processes through 
studies of resolved stars in the halo: it provides an external perspective of the nearest large galaxy, where all of M31's stars are at about the same distance from us, in contrast to the situation in our own Galaxy, which allows us to obtain a relatively complete picture of its stellar halo.

Past observational studies of M31's halo through large photometric and spectroscopic surveys of bright RGB/AGB stars - e.g., the PAndAS survey using CFHT/MegaCam (McConnachie et al. 2009), the SPLASH survey using KPNO-Mayall/ MOSAIC, Keck/DEIMOS and Subaru/Suprime-Cam (Guhathakurta et al. 2005; Gilbert et al. 2006; Tanaka et al. 2010)—have revealed several characteristic properties of M31's stellar halo, some similar to and some different from those of the MW halo. The similarities to the MW halo are in M31's outer halo, which has a power-law surface brightness profile, low stellar density, and metal-poor stars (Guhathakurta et al. 2005; Kalirai et al. 2006; Gilbert et al. 2012, 2014; Ibata et al. 2014), whereas the differences are seen in the inner halo of M31, which has metal-rich and intermediate-age populations in contrast to the largely metal-poor and old halo stars in the MW. The presence of substructures in M31's halo, such as the Giant Southern Stream, and the nonisotropic distribution of satellites (Ibata et al. 2013) are also seen in the MW.

These observational results for M31's halo, which provide us with a new view of an ancient stellar halo compared to the MW halo, have motivated many subsequent simulations of galaxy formation. Indeed, in $\Lambda$-dominated cold dark matter models, each disk galaxy has been developed through a different assembly and evolutionary path: the collapse epoch, star formation history, and assembly rate of subsystems are not the same from one halo to another, and each stellar halo is thus expected to have a different morphology (e.g., Bullock \& Johnston 2005; Cooper et al. 2010). Further observational studies of M31's halo will thus be of great importance for gaining new insights into the formation process of its present structure, especially the origin of the differences relative to the MW halo.

In this work, we report our new observations of M31's halo using Subaru/Hyper Suprime-Cam (HSC). HSC is a new primefocus camera with a 1.5 diameter field of view on the Subaru Telescope (Miyazaki et al. 2012, 2017; Furusawa et al. 2017; Kawanomoto et al. 2017; Komiyama et al. 2017).

This combination of HSC and Subaru thus allows us to survey large areas of M31's halo with only a small number of pointings and to explore much deeper domains of the halo, including faint red giants and horizontal-branch stars, than earlier studies based on $4 \mathrm{~m}$ telescopes have revealed. This work will focus on the detailed structure and stellar populations of the Northwestern (NW) Stream, which is full of substructures, including dips and gaps, which may have been induced by interaction with orbiting subhalos (Carlberg et al. 2011; Carlberg 2012). Our HSC observations thus provide new insights into the origin of these halo structures.

The paper is organized as follows. In Section 2, we describe our observations of M31's halo with Subaru/HSC and the method for data analysis. Our target fields are those covering large parts of the NW Stream (Figure 1). In Section 3, the spatial distribution of halo stars in our survey regions and their distribution on color-magnitude diagram (CMD) are presented. Section 4 is devoted to the detailed analysis of stellar populations inside/outside the NW stream, their distance distribution, and the three-dimensional structure of the NW

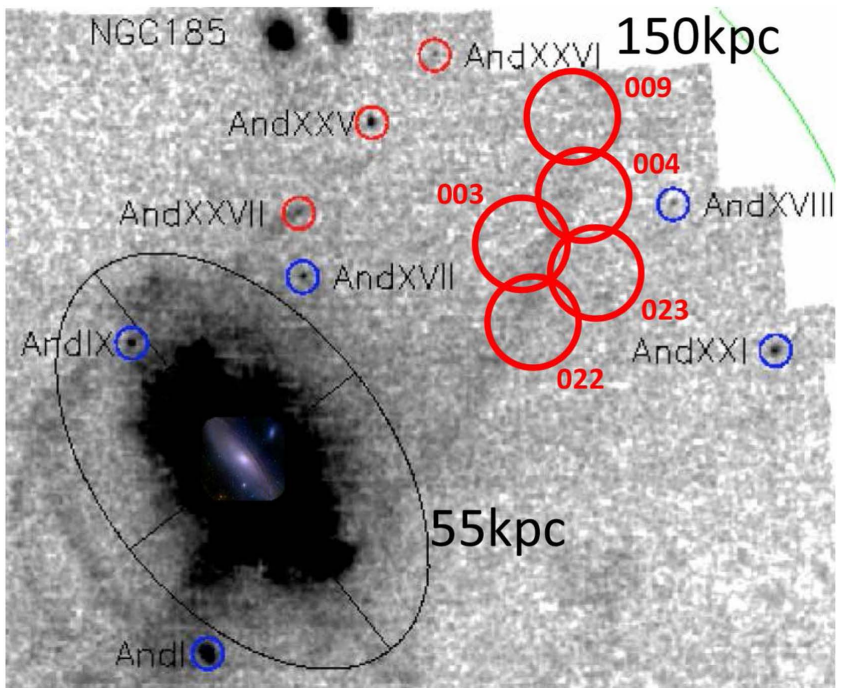

Figure 1. HSC deep survey fields (numbered large red circles) overlaid on Figure 1 of Richardson et al. (2011) showing the central image of M31 and surrounding star count map (grayscale) with some dwarf satellites indicated (small blue and red circles).

stream. In Section 5, we discuss the nature of the NW stream in comparison to the distribution of globular clusters and a recent numerical simulation of the stream. Finally, we present our conclusions in Section 6.

\section{Data and Method}

\subsection{HSC Observations}

We obtained HSC $g$ - and $i$-band images over nine nights in 2014 and six nights in 2015. The observing conditions were variable, and we devoted observing time in relatively good condition (i.e., fair transparency and better seeing FWHM than 0 .' 8 ) to a deep survey in the halo of M31, which we report on in this paper. Figure 1 shows the target fields, which consist of five contiguous pointings (Fields 003, 004, 009, 022, and 023), covering a $9.2 \mathrm{deg}^{2}$ area in the halo of M31. In each field, dithering with radius of 2 arcmin was performed to cover the gaps between CCDs. During the observation, the transparency was good to fair, but the seeing FWHM varied between 0." 45 and 0 ." 8 . Total exposure times for each field were 80 minutes and 80-128 minutes in the $g$ and $i$ bands, respectively.

We also obtained short exposure images for the five target fields using the narrowband filter NB515, which has a bellshaped transmission curve centered on $515 \mathrm{~nm}$ with a bandwidth (FWHM) of $7.7 \mathrm{~nm}$ and samples $\mathrm{Mg} b$ features of late-type stars (see Figure 2). It is equivalent to the DDO51 filter, which is useful for distinguishing between giant and dwarf stars based on the depth of stellar surface gravity sensitive spectral absorption features (Majewski et al. 2000; Morrison et al. 2000). Although the NB515 exposure times are as short as 16 minutes for each field, the data are deep enough to separate the bright RGB stars of M31 from the foreground main-sequence (MS) dwarf stars in the MW.

The details of the observations are summarized in Table 1, and the filter response curves are plotted in Figure 2.

\subsection{Reduction and Photometry}

The HSC data are processed with hscPipe v4.0.1 (Bosch et al. 2017), a branch of the Large Synoptic Survey Telescope 


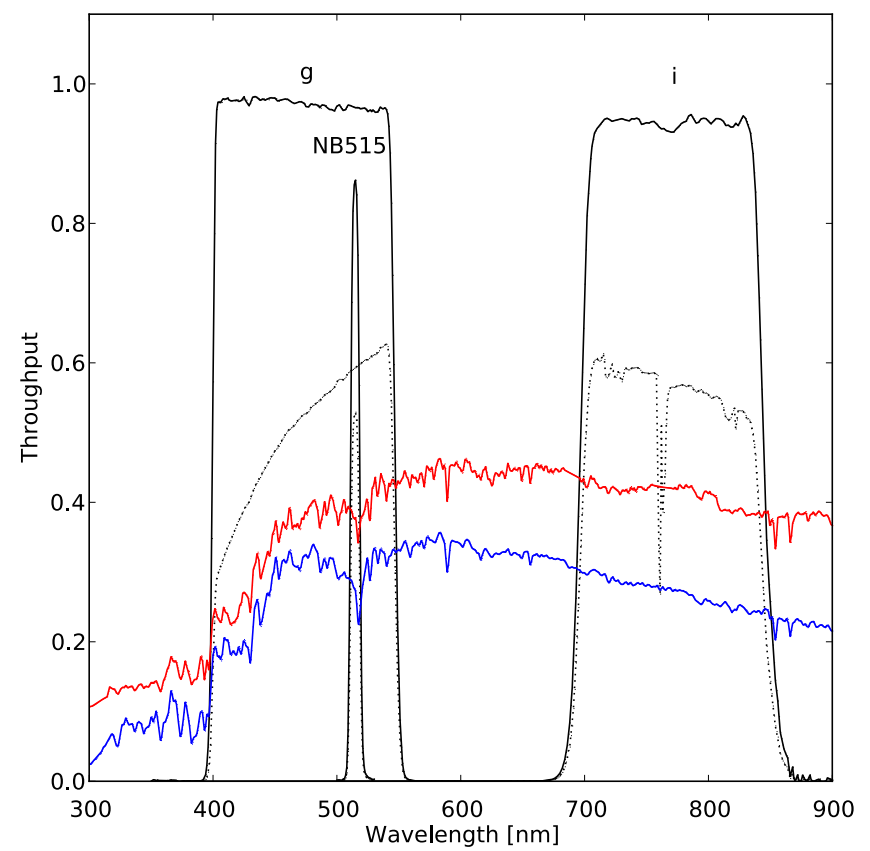

Figure 2. Response curves of filters used in the survey. Solid lines show the response curves for the filters themselves, and the dotted lines show the total response. The spectra of K1V (blue) and K1III (red) stars from the Pickles stellar spectral flux library (Pickles 1998) are overlaid. Note that both spectra are averaged for five bins (i.e., $2 \mathrm{~nm}$ ), and the spectrum of the K1III star is shifted upwards for clarity.

Table 1

The Details of the Observation

\begin{tabular}{|c|c|c|c|c|}
\hline Field & R.A., Decl. (J2000) & Filter & Exp. Time & Seeing FWHM \\
\hline 003 & $\begin{array}{l}00^{\mathrm{h}} 16^{\mathrm{m}} 31^{\mathrm{s}} .7 \\
+44^{\circ} 43^{\prime} 30^{\prime \prime}\end{array}$ & $\begin{array}{c}g \\
i \\
N B 515\end{array}$ & $\begin{array}{c}12 \times 400 \mathrm{~s} \\
31 \times 240 \mathrm{~s} \\
4 \times 240 \mathrm{~s}\end{array}$ & $\begin{array}{c}0 . ! 7-0 . ! ~ 8 \\
0 . ! 5-0 . ! 8 \\
0 . ! 6-0 . ! 75\end{array}$ \\
\hline 004 & $\begin{array}{l}00^{\mathrm{h}} 10^{\mathrm{m}} 04^{\mathrm{s}} .7 \\
+45^{\circ} 27^{\prime} 47^{\prime \prime}\end{array}$ & $\begin{array}{c}g \\
i \\
N B 515\end{array}$ & $\begin{array}{c}12 \times 400 \mathrm{~s} \\
30 \times 240 \mathrm{~s} \\
4 \times 240 \mathrm{~s}\end{array}$ & $\begin{array}{c}0 . ! 6-0 . " 8 \\
0 . ! 45-0 .{ }^{\prime \prime} 8 \\
0 . ! 55-0 .{ }^{\prime \prime} 6\end{array}$ \\
\hline 009 & $\begin{array}{l}00^{\mathrm{h}} 10^{\mathrm{m}} 24^{\mathrm{s}} .5 \\
+46^{\circ} 49^{\prime} 07^{\prime \prime}\end{array}$ & $\begin{array}{c}g \\
i \\
N B 515\end{array}$ & $\begin{array}{c}12 \times 400 \mathrm{~s} \\
20 \times 240 \mathrm{~s} \\
4 \times 240 \mathrm{~s}\end{array}$ & $\begin{array}{c}0 .{ }^{\prime \prime} 65-0 . \\
0 .{ }^{\prime \prime} 6-0 . \\
0 . \\
0.55-0 .\end{array}$ \\
\hline 022 & $\begin{array}{l}00^{\mathrm{h}} 16^{\mathrm{m}} 04^{\mathrm{s}} .2 \\
+43^{\circ} 22^{\prime} 15^{\prime \prime}\end{array}$ & $\begin{array}{c}g \\
i \\
N B 515\end{array}$ & $\begin{array}{l}12 \times 400 \mathrm{~s} \\
32 \times 240 \mathrm{~s} \\
4 \times 240 \mathrm{~s}\end{array}$ & $\begin{array}{c}0 . .5-0 .{ }^{\prime \prime} 8 \\
0 . " 45-0 . ! 8 \\
0 . ! 85-0 . ! 95\end{array}$ \\
\hline 023 & $\begin{array}{l}00^{\mathrm{h}} 09^{\mathrm{m}} 45^{\mathrm{s}} .8 \\
+44^{\circ} 06^{\prime} 28^{\prime \prime}\end{array}$ & $\begin{array}{c}g \\
i \\
N B 515\end{array}$ & $\begin{array}{l}12 \times 400 \mathrm{~s} \\
30 \times 240 \mathrm{~s} \\
4 \times 240 \mathrm{~s}\end{array}$ & 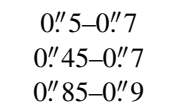 \\
\hline
\end{tabular}

pipeline (Ivezic et al. 2008; Juric et al. 2015) calibrated against Pan-STARRS1 (PS1) photometry and astrometry (Schlafly et al. 2012; Tonry et al. 2012; Magnier et al. 2013). The hscPipe is a standard data reduction pipeline optimized for the data from widefield mosaic CCD cameras in general and HSC in particular. Each CCD frame is calibrated against PS1 in the course of single frame reduction (i.e., bias subtraction, correction for cross-talk, cosmic ray removal, flat fielding, sky subtraction). Then, all of the frames are co-added into a large image based on the mosaicking solution, which is calculated using all of the frames in each band. Finally, objects are detected, and their parameters such as magnitudes, positions, sizes, etc. are measured.
The resultant photometric catalogs in all three bands are matched with a matching radius of 0.15 . In this study, we analyze those objects that are classified as point sources (i.e., extendedness $=0$ in the hscPipe output catalog) in both $g$ and $i$ bands, and PSF magnitudes are used throughout this study except in Section 5.1. Note that the NB515 data were calibrated to the $g$ band via hscPipe, and the NB515 magnitude may be offset systematically from the correct value by $\leqslant 0.1 \mathrm{mag}$, but the absolute value is not important for our purposes.

Our survey is substantially deeper than previous groundbased surveys of M31. We assess how deep our data go by measuring the detection completeness and estimating the extinction by interstellar dust, as described below, before we start further analysis.

The detection completeness is estimated using a PSF model obtained by the hscPipe software for every $4000 \times 4000$ pixel grid, which is referred to as a "patch" in hscPipe. A set of 500 artificial stars based on the PSF model and a specific apparent magnitude is embedded in a patch, and the hscPipe object detection algorithm is applied. This process is repeated in steps of $0.25 \mathrm{mag}$, and the completeness, the fraction of artificial stars that are detected, is obtained for all patches. Figure 3 shows the apparent magnitude that corresponds to $50 \%$ completeness in the $g, i$, and NB515 bands over our survey field. The mean magnitudes of $50 \%$ completeness in the $g, i$, and NB515 bands are calculated to be 26.31, 25.69, and 24.71 mag, respectively, indicating that our survey is substantially deeper than the previous ground-based studies. Field 023 is the deepest among the survey fields in the $g$ and $i$ bands, and the northern part is relatively shallow compared to the southern part, likely due to the unstable weather and variable seeing conditions during the observations. Patches that contain bright stars are shallower than neighboring patches, which indicate that the completeness is degraded by the large halo of a bright star. Therefore, areas occupied by large halos around bright stars are masked and excluded from the subsequent analysis. The NB515 band imaging in the northern and middle parts is deeper than that in the southern part. This is due to variable seeing conditions between the different pointings (see Table 1).

The Galactic extinction is corrected using the new estimate from Schlafly \& Finkbeiner (2011), which is based on the dust map by Schlegel et al. (1998). Figure 4 shows the twodimensional $E(B-V)$ map for our survey field, where the $E$ $(B-V)$ values are taken from the NASA/IPAC Infrared Science Archive. ${ }^{20}$ The variation of $E(B-V)$ across our survey field is clearly seen in the sense that the northernmost part (Field 009) suffers from heavy extinction while the majority of the four other fields are less affected. Therefore, the extinction correction should be made star by star according to the position on the sky. The mean extinctions in the $g, i$, and NB515 bands are calculated to be $0.27,0.14$, and $0.24 \mathrm{mag}$, respectively. The accuracy of the estimate of extinction based on this method is discussed in Section 4.4.

The spatially varying completeness and extinction resulted in inhomogeneity in the survey depth (i.e., limiting magnitude) across the field, in the sense that the southern part (Fields 022 and 023) is the deepest, and the survey depth gets slightly worse at the middle part (Fields 003 and 004) and significantly degraded at the northern part (Field 009) for the $g$ and $i$ bands, while the opposite trend is seen in the NB515 band. We will

\footnotetext{
${ }^{20}$ http://irsa.ipac.caltech.edu/applications/DUST/
} 


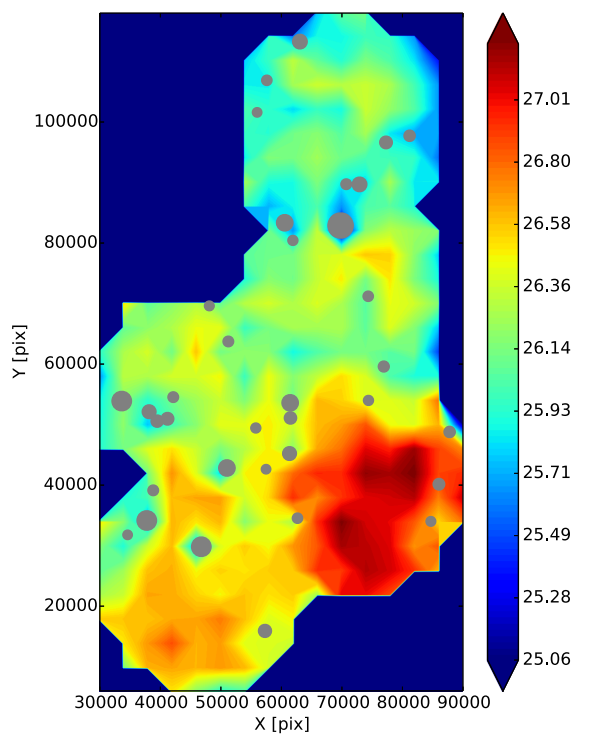

(a) $g$ band

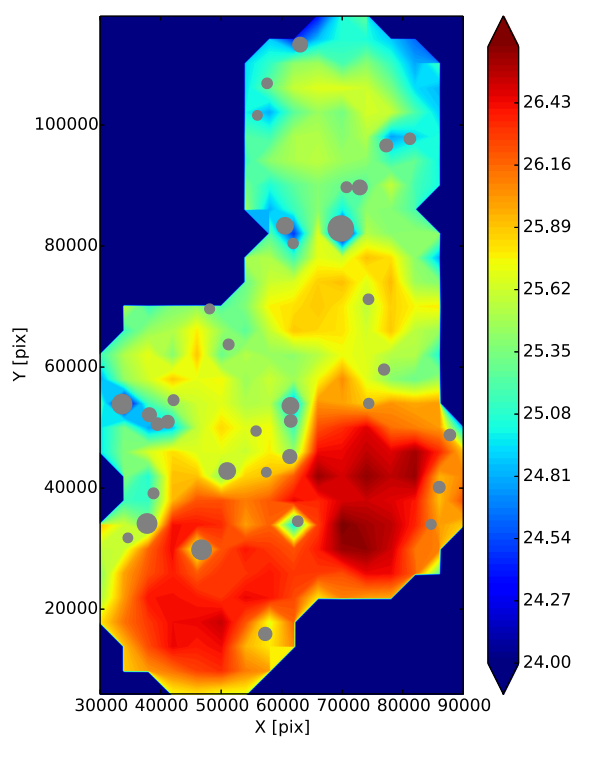

(b) $i$ band

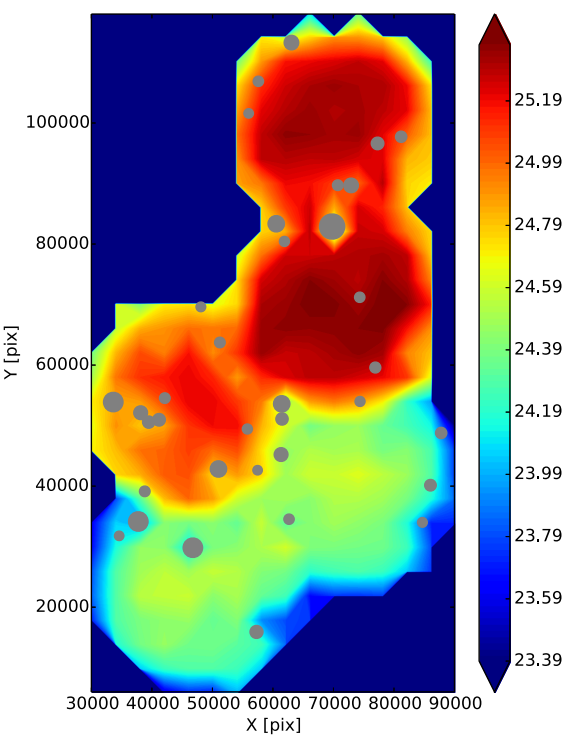

(c) NB515 band

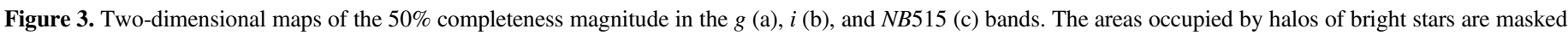
and shown by the gray circles.

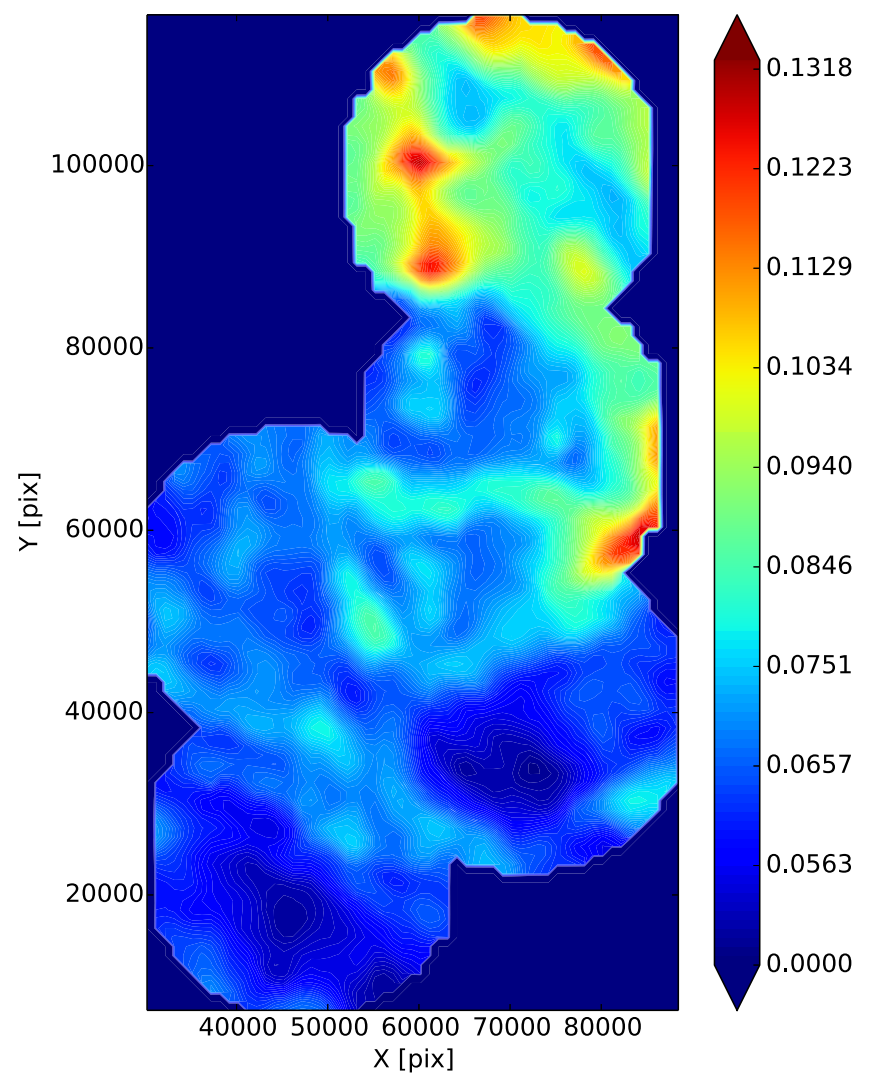

Figure 4. Two-dimensional $E(B-V)$ map for our survey field.

take the spatial variation into account in the following analysis. Nonetheless, our data are considerably deep and bring a wealth of information to understand the nature of the M31 halo.

\subsection{Color-Magnitude Diagram}

Figure 5 is the extinction-corrected CMD of 401,834 catalogued stellar objects down to $i=27.0$ mag in our survey

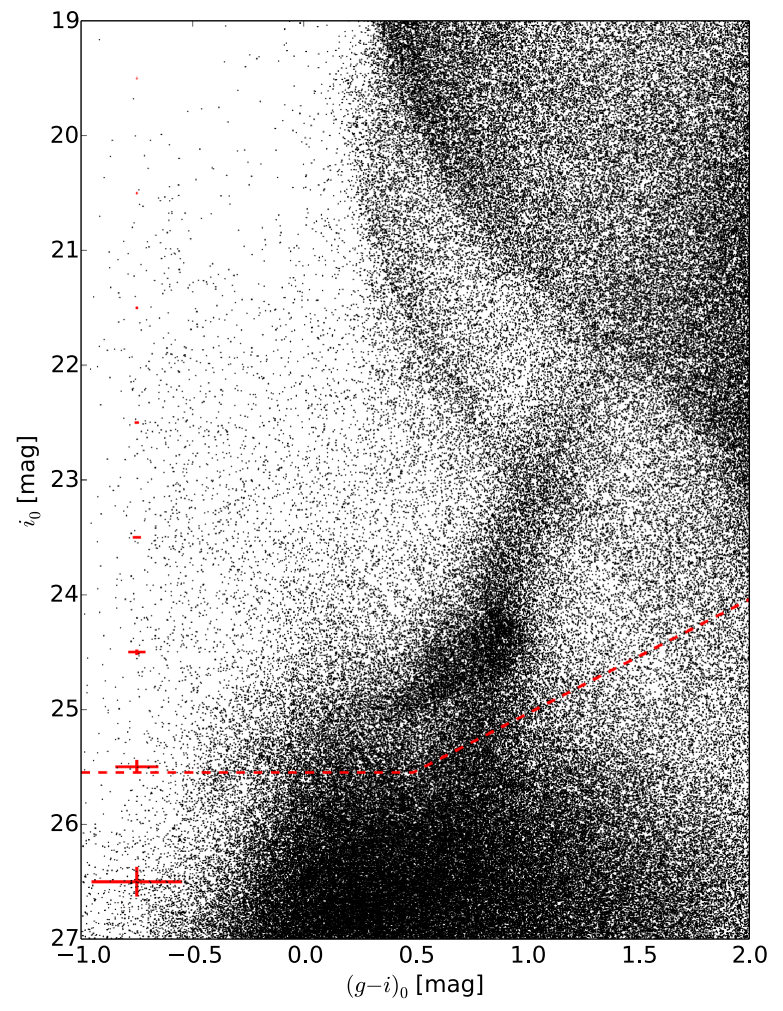

Figure 5. Color-magnitude diagram of catalogued stellar objects in our survey field. The photometric error in each band, which is the mean value of every 1 mag bin, is calculated, and the errors in the $i_{0}$ magnitude and color (calculated for $\left.(g-i)_{0}=1.0\right)$ are plotted as red crosses. The $50 \%$ completeness limit, which is the mean value over the survey field, is plotted as a red dashed line.

fields. The photometric error in each band, which is the mean value of every $1 \mathrm{mag}$ bin, is calculated, and the errors in the $i_{0}$ magnitude and color (calculated for $\left.(g-i)_{0}=1.0\right)$ are plotted as red crosses in the figure. The $50 \%$ completeness limit, which is the mean value over the survey field, is plotted as a red dashed line. 


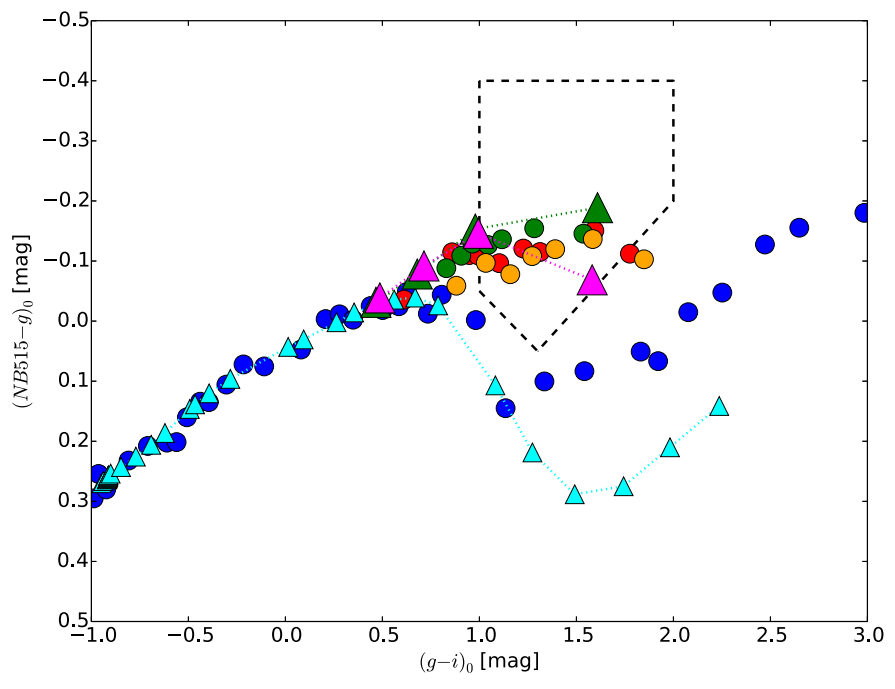

(a)

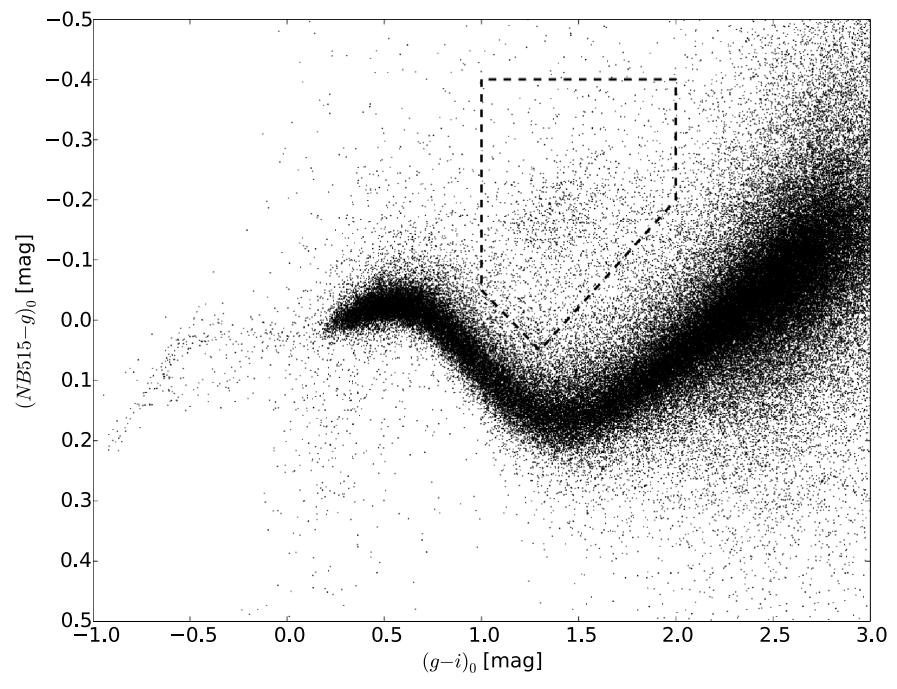

(b)

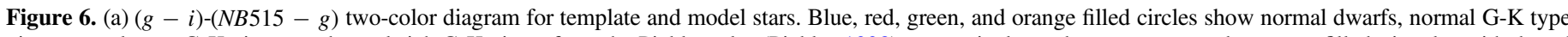

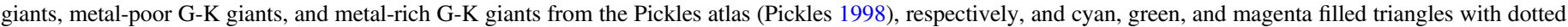

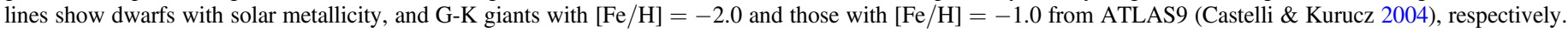

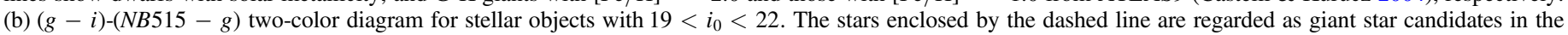
M31 halo (NBGs).

The CMD clearly shows the characteristic features: the dwarf stars of the MW disk lie on the brightest part of the CMD, extending from $(g-i)_{0} \simeq 0.3$ and $i_{0} \simeq 19$ to the redder and fainter part of the CMD. A narrow but significant sequence seen at $\sim 1$ mag fainter from the distribution of the MW disk stars corresponds to a diffuse stellar stream in the MW halo, which was reported by Martin et al. (2014). The RGB in the M31 halo is seen as a broad sequence that is perpendicular to the above-mentioned MW stream and crossing at $(g-i)_{0} \simeq 1.1$ and $i_{0} \simeq 23$. We also note that a significant red clump feature is seen at $(g-i)_{0} \simeq 0.8$ and $i_{0} \simeq 24.5$, and a diffuse but distinct blue horizontal branch (BHB) is also seen at $(g-i)_{0} \simeq-0.3$ and $i_{0} \simeq 25.5$. We call those objects found in $0.3<(g-i)_{0}<1.0,24.0<i_{0}<25.1$ and $g_{0}<25.6$ the red clump population (RC) in the following analysis. Below $i_{0}=26$, the photometric error becomes large, and it is difficult to distinguish characteristic features such as the sub-giant branch and MS stars in the M31 halo.

\subsection{NB515 and Two-color Diagram}

To extract secure M31 RGB stars behind foreground Galactic dwarf stars, we use a narrowband filter, NB515, in combination with the $g$ - and $i$-band filters. This filter has been designed by our team for this purpose, and we have already performed a test to distinguish M31's RGB/AGB stars from foreground Galactic dwarfs using the prototype NB515 filter in the Suprime-Cam and confirmed its validity. As shown in Figure 2, NB515 is sensitive to the $\mathrm{MgH}+\mathrm{Mg} b$ absorption features around $515 \mathrm{~nm}$, which is strongly dependent on surface gravity (Majewski et al. 2000 and references therein). By measuring the absorption strength of this feature with the NB515 filter, one can discriminate K giants in the halo of M31 from $\mathrm{K}$ dwarfs in the disk of the MW with the same apparent magnitude. This is illustrated in Figure 2, where the spectra of K1V (blue) and K1III (red) stars from the Pickles stellar spectral flux library (Pickles 1998) are well discriminated by the absorption feature, i.e., the NB515 $-g$ color, in the sense that the $N B 515-g$ color at a fixed $g-i$ color is larger for dwarfs and smaller for giants.

We investigate how dwarfs and giants are distributed in the two-color diagram, making use of template spectra in Figure 6(a). We use the Pickles stellar spectral flux library (Pickles 1998) and ATLAS9 stellar atmosphere models (Castelli \& Kurucz 2004), and the $g-i$ and NB515 $-g$ colors are calculated by convolving the system throughput as shown in Figure 2. Blue, red, green and orange filled circles show normal dwarfs, normal G-K type giants, metal-poor G-K giants, and metal-rich G-K giants from the Pickles atlas, respectively, and cyan, green, and magenta filled triangles with dotted lines show dwarfs with solar metallicity, and G-K giants with $[\mathrm{Fe} / \mathrm{H}]=-2.0$ and those with $[\mathrm{Fe} / \mathrm{H}]=-1.0$ from ATLAS9, respectively. The suggested models ${ }^{21}$ for the dwarfs of ATLAS9 are used for dwarfs with solar metallicity, while $T_{\text {eff }}$ and $\log (g)$ are chosen from suggested models of G-K giants, and those with different metallicities are used to calculate $g-i$ and NB515 $-g$ colors. The figure indicates that giants can clearly be discriminated from dwarfs for $g-i>1.0$. Our criteria to select giants, which are represented by the dashed line, fairly well enclose giants, except for normal to metal-rich $\mathrm{K} 5$ giants.

Figure 6(b) shows the two-color diagram for stellar objects with $19<i_{0}<22$ in our survey field. The thick "V" -shape sequence is composed of MS dwarf stars of the MW. On the other hand, the giant stars in the M31 halo populate around our criteria, which are shown by the dashed line. The criteria are set reasonably to select giants in the M31 halo while eliminating contaminations from MS dwarfs. In this study, we regard those objects enclosed by the dashed line in Figure 6 to be giant star candidates in the M31 halo and call them narrowband-selected giants (NBGs).

\footnotetext{
${ }^{21}$ http://www.stsci.edu/hst/observatory/crds/castelli_kurucz_atlas.html
} 


\section{Stellar Populations in the Survey Fields}

\subsection{Spatial Distribution}

Figure 7 shows the distributions of NBGs with $19<i_{0}<23$ and RCs. A distinct stream feature, which is already shown to exist by the PAndAS survey and called the NW Stream (McConnachie et al. 2009; Richardson et al. 2011), is clearly seen in the figure. Our data reveal much more detailed properties of the NW Stream; the stars composing the stream are not uniformly distributed and instead show a clumpy distribution.

Besides the NW Stream, the surface densities of NBGs and $\mathrm{RCs}$ in the off-stream region seem to be higher at the southern part compared to the northern part. This trend may be due to the different limiting magnitudes among the survey field, in particular for the RC population. We calculate the 50\% completeness contour to detect the RC population (i.e., those stars with $0.3<(g-i)_{0}<1.0, \quad 24.0<i_{0}<25.1$, and $\left.g_{0}<25.6\right)$, which is plotted as the orange dotted line in Figure 7(b). The figure indicates that most of the survey field is more than $50 \%$ complete, except for the periphery of the field of view of HSC pointing at the northern part, where the extinction is heavy. We therefore conclude that this decreasing trend toward the north is real. The same trend is also seen for NBGs, supporting the fact that this trend is real.

In the following, we analyze the data divided into four regions, Stream North/South and Off-Stream North/South, and investigate the stellar populations in the four regions separately. The boundaries are shown by the red dashed lines, and each region is labeled in Figure 7. The boundary between north and south $(\delta=44.8)$ is set so that the number of stars is almost the same between north and south in the off-stream region.

\subsection{CMDs of the Four Regions}

Figure 8 shows the CMDs of the four regions. In panel (a), the overall CMD for each region is plotted with the $50 \%$ completeness limit in the $g$ and $i$ bands, which is the mean value in each region, and the mean error bars for every $1 \mathrm{mag}$ interval are plotted as a red dashed line and red crosses, respectively, in the respective figure. Panel (b) shows only NBGs, and panel (c) shows the zoomed-in density map of the $\mathrm{RC}$ regions. The corresponding $50 \%$ completeness limit in NB515, which is translated from the narrowband selection criteria, is plotted as a blue dashed line in panel (b). Note that the $50 \%$ completeness limit in NB515 is only effective for NBGs (red points).

The CMD of Stream North clearly shows a narrow RGB, populated RC, and a hint of a distinct BHB, although the survey depth is shallow, suggesting that the old and metal-poor population dominates in Stream North. The peak of the RC is found at $(g-i)_{0} \simeq 0.6$ and $i_{0} \simeq 24.8$ from panel (c).

On the other hand, the CMD of Stream South shows a different appearance: wider RGB, more abundant RC with multiple peaks, and a tight BHB sequence. The differences, such as the tight BHB sequence, may be explained by the difference of the limiting magnitude, but additional stellar populations, which are different from those found in the Stream North region, are strongly suggested to exist in the Stream South region. Two distinct peaks of $\mathrm{RC}$ are found at

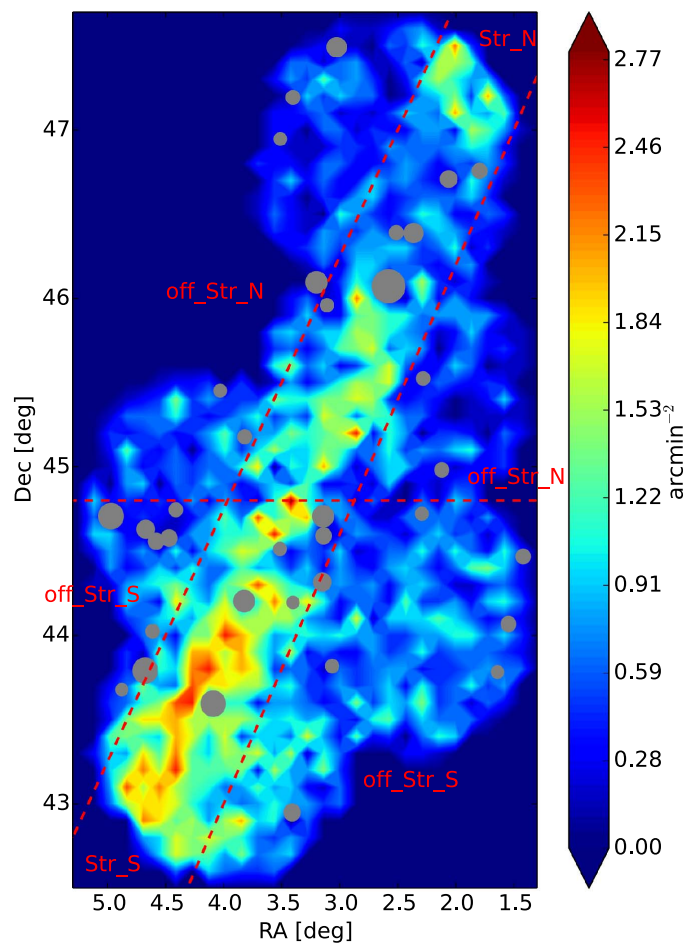

(a) NBG

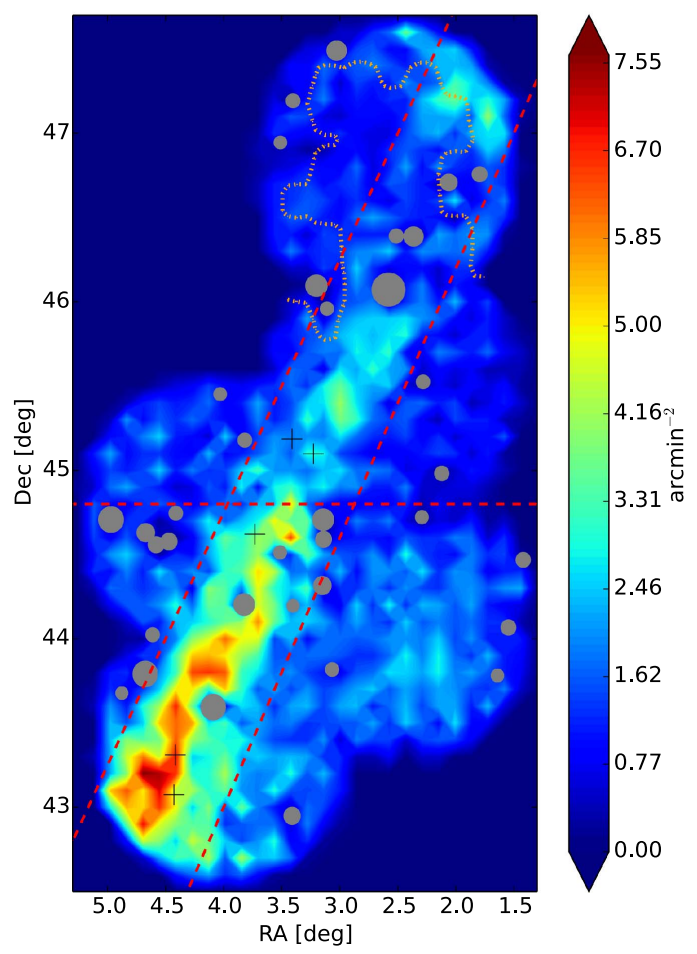

(b) $\mathrm{RC}$

Figure 7. Surface number density maps of NBGs stars (a) and RCs (b). Areas near bright stars where the object detection is not well carried out are masked with gray circles. The boundaries between Stream/Off-Stream North/South regions are shown by the red dashed lines, and each region is labeled in panel (a). In panel (b), the region where $50 \%$ completeness is achieved at magnitudes of $i_{0}=25.1 \mathrm{mag}$ and $g_{0}=25.6 \mathrm{mag}$, which is the fainter boundary of RC selection, is shown by the orange dotted line, indicating that most of the survey field is more than $50 \%$ complete to detect RC stars. Crosses represent the five globular clusters reported by Huxor et al. (2014) in the survey field. 


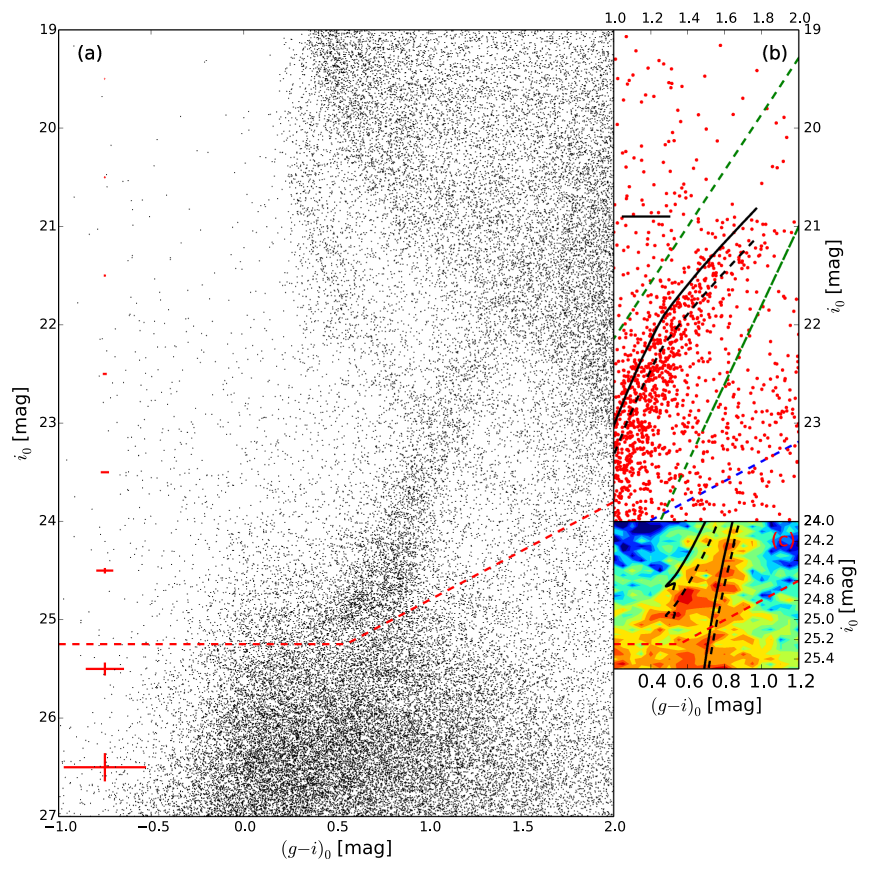

(1) Stream North

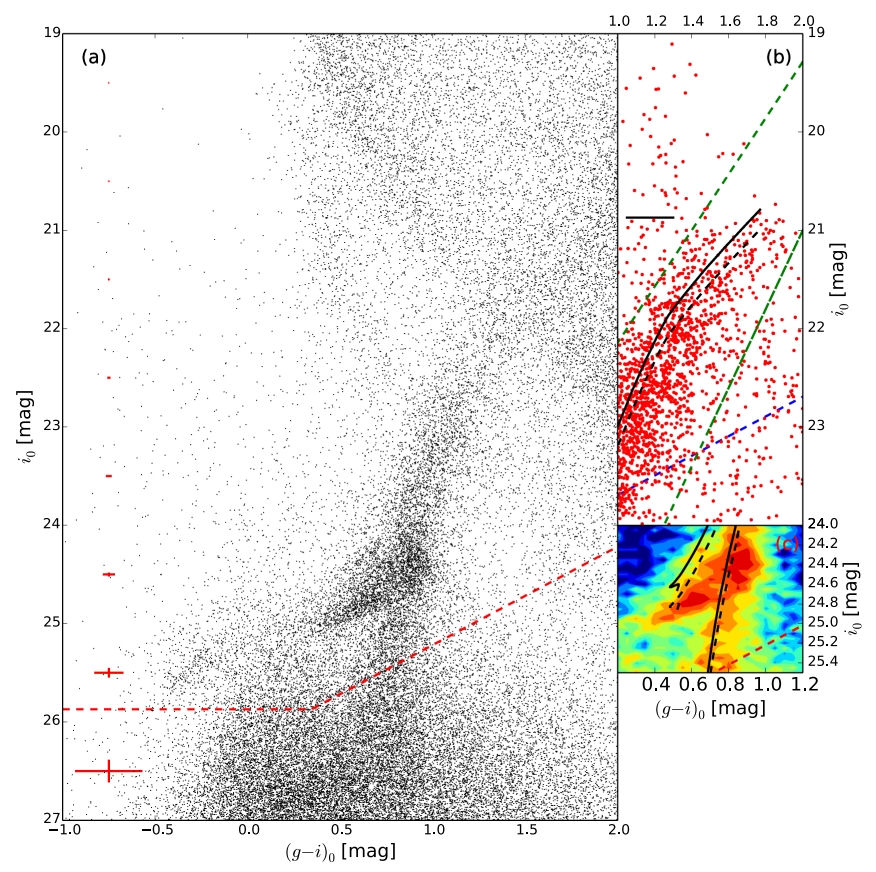

(3) Stream South

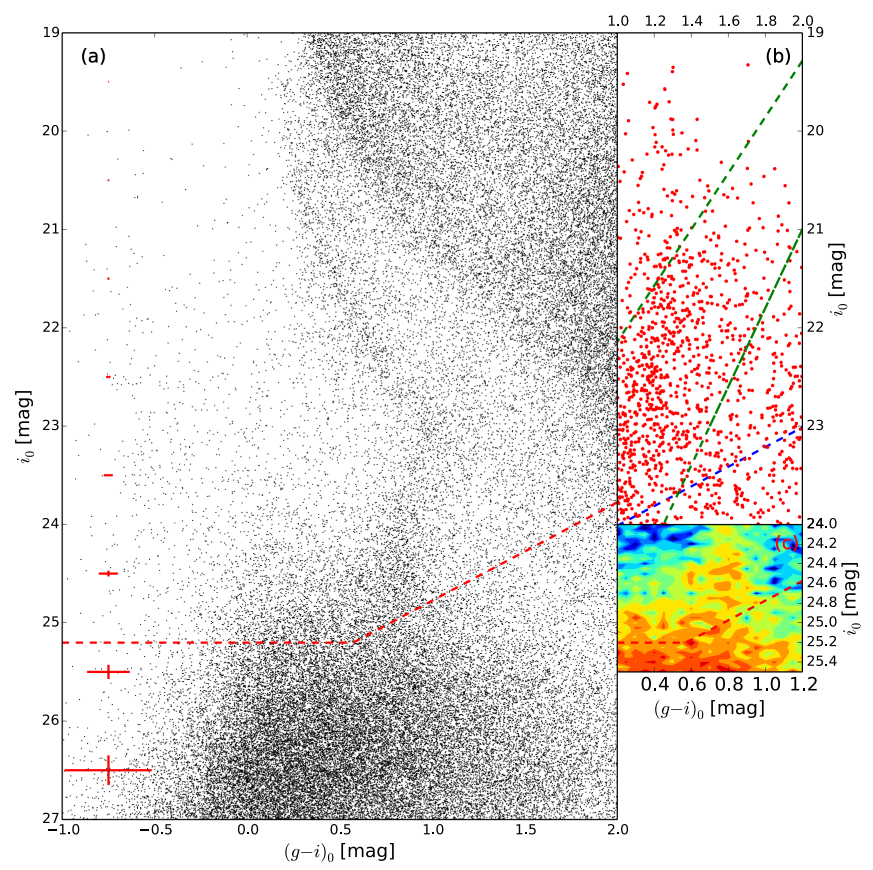

(2) Off-Stream North

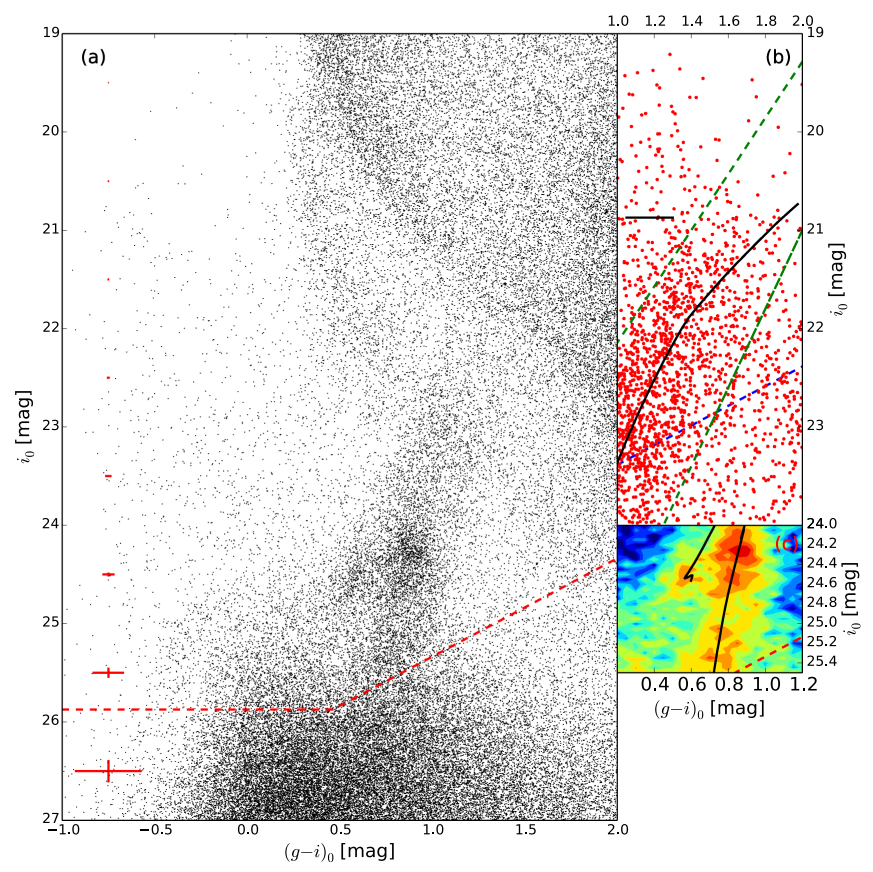

(4) Off-Stream South

Figure 8. CMDs of the four regions in our survey field. From top left to bottom right, the CMDs of (1) Stream North, (2) Off-Stream North, (3) Stream South, and (4) Off-Stream South are plotted. For each of the subfigures (1)-(4), panel (a) shows the overall CMD. The 50\% completeness limit in the $g$ and $i$ bands, which is the mean value in each region, and the mean error bars for every 1 mag interval are plotted as a red dashed line and red crosses, respectively. Panel (b) shows the zoomedin view of the RGB region with the NBGs plotted in red. The corresponding 50\% completeness limit in NB515 is plotted as a blue dashed line. The RGB selection used to determine the TRGB is plotted as green dashed lines, and the TRGB magnitude is indicated by the horizontal black bar. The best-fit isochrone is plotted as the thick black line. Panel (c) shows the zoomed-in density map of the RC region, together with the best-fit isochrone, with the distance modulus determined using the TRGB method (thick black line) and that with the distance modulus from the TRGB (dashed black line) increasing by 0.3 (Stream North) and 0.2 (Stream South).

$(g-i)_{0} \simeq 0.6$ and $\quad i_{0} \simeq 24.8$ and $\quad$ at $\quad(g-i)_{0} \simeq 0.8$ and $i_{0} \simeq 24.4$.

The CMD of Off-Stream South also shows a wider RGB and populated RC, but the morphology of RC is different from that of Stream South/North. In addition, no tight sequence of the BHB is observed in Off-Stream South. The peak of the RC is found at $(g-i)_{0} \simeq 0.8$ and $i_{0} \simeq 24.3$, which seems to be coincident with one of the RC peaks found for Stream South. This finding indicates that the CMD of Stream South can be reproduced by a combination of CMDs of Stream North and Off-Stream South. 
Although the survey depth of the Off-Stream North region is shallow, its CMD is different from that of Off-Stream South (e.g., populated $\mathrm{RC}$ and $\mathrm{RGB}$ ), suggesting the presence of a diffuse but significant substructure in the Off-Stream South region. We also note that the number of NBGs, which are not affected by the survey depth, is smaller compared to that found in each of the other three regions (see panels (b)). These findings lead to the idea that the Off-Stream North region is genuinely the region with no substructure, i.e., a part of the smooth halo of M31. The loose RGB sequence and the absence of RCs observed for Off-Stream North also support this idea because stars in the halo are widely distributed along the line-of-sight direction and the features in the CMD, such as the RGB, RC, and $\mathrm{BHB}$, are smoothed out along the magnitude axis.

The conclusions of this section are as follows. In addition to the known NW Stream, a diffuse substructure is likely to exist in the southern part of our survey field. In the Stream South region, the NW Stream and diffuse substructure overlap. In contrast, the Off-Stream North region seems to be free of substructures and represents a genuine smooth halo of M31. In the following analysis, we regard Stream North, Off-Stream South, and OffStream North as representative of the NW Stream, the diffuse substructure, and the smooth halo, respectively.

\section{Basic Properties of Stellar Populations}

\subsection{Tip of the Red Giant Branch (TRGB)}

As shown in Figure 5, the bright part of the RGB of M31's halo stars is merged into the higher density sequence consisting of numerous MW MS stars. We can separate the RGB stars from those overlapping MW MS stars by making use of NB515 data. The TRGB is now visible by plotting NBGs in the CMDs of Figure 8, panels (b).

Following the recent technique to determine the TRGB magnitude based on the Bayesian approach (e.g., Conn et al. 2011; Tollerud et al. 2016; Tanaka et al. 2017), we attempt to determine the TRGB magnitude from NBGs, which are supposed to be a clean sample of RGBs in principle, for the four regions separately. The method we adopt is a maximumlikelihood estimation based on the Markov Chain Monte Carlo (MCMC) algorithm, which is described in detail by Tanaka et al. (2017). In this study, we assume the model luminosity function (LF) to be

$$
\phi\left(m \mid m_{\mathrm{TRGB}}, a, b\right)=e^{a\left(m-m_{\mathrm{TRGB}}\right)}+b, \quad\left(\text { for } m \geqslant m_{\mathrm{TRGB}}\right)
$$

$$
=b, \quad\left(\text { for } m<m_{\mathrm{TRGB}}\right),
$$

where $m_{\mathrm{TRGB}}$ is the TRGB magnitude. We also assume $100 \%$ completeness for our NBG sample. The prior distribution assumed for $m_{\mathrm{TRGB}}$ is a normal distribution with the mean, which is estimated from the edge-detection algorithm (Sakai et al. 1996) applied to the data in each region, and $\sigma=$ $0.5 \mathrm{mag}$, and that for $a$ and $b$ is a uniform distribution between 0 and 2. The number of chains used in this study is 4 . We also apply a color cut, $-0.35\left(i_{0}-23\right)+0.7 \leqslant(g-i)_{0} \leqslant$ $-0.25\left(i_{0}-23\right)+1.5$, to eliminate those stars that deviate from RGBs. As shown in panels (b) of Figure 8, this color cut, which is represented by the green dashed lines, clearly excludes outliers and distinguishes the TRGB.

Figure 9 shows the result. The blue histogram shows the number distribution of NBGs, and the dashed green line shows

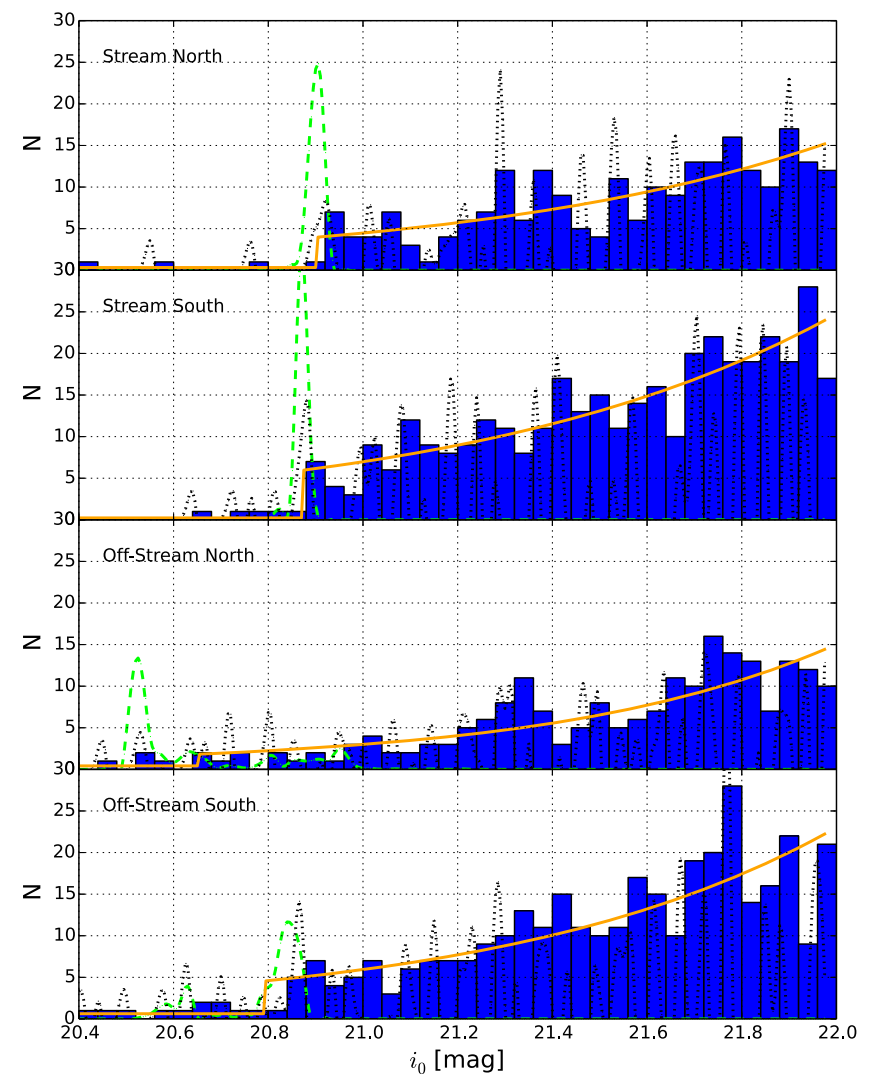

Figure 9. The blue histogram shows the number distribution of NBGs, and the dashed green line shows the frequency of $m_{\text {TRGB }}$ obtained from 20,000 MCMC runs after 80,000 unused runs. The orange line is the model LF, $\phi\left(m_{n} \mid m_{\mathrm{TRGB}}, a, b\right)$, for which mean values of $m_{\mathrm{TRGB}}, a, b$ are used. For comparison, we also plot the edge-detection filter described by Sakai et al. (1996) as black dotted lines.

the frequency of $m_{\mathrm{TRGB}}$ obtained from 20,000 MCMC runs after 80,000 unused runs. The orange line is the model LF, $\phi\left(m_{n} \mid m_{\mathrm{TRGB}}, a, b\right)$, for which mean values of $m_{\mathrm{TRGB}}, a, b$ are used. The figure clearly shows that the model LF fits the observed distribution of NBGs reasonably well and that $m_{\mathrm{TRGB}}$ is well-determined for the Stream North and South regions and fairly determined for Off-Stream South. On the other hand, the determination of $m_{\mathrm{TRGB}}$ is poor for Off-Stream North since the number of NBGs found for this region is small, and it is difficult to recognize the sharp rise of LF at $m_{\mathrm{TRGB}}$. For comparison, we also plot the edge-detection filter described by Sakai et al. (1996) as the black dotted lines, suggesting that both estimates are consistent with each other.

Table 2 summarizes the distribution of $m_{\mathrm{TRGB}}$. We take the peak value as the representative TRGB magnitude and the $68 \%$ interval of the distribution around the peak as the error. We obtain the TRGB magnitudes as $i_{0}=20.90 \pm 0.02$, $20.87 \pm 0.02$, and $20.84 \pm 0.03$ mag for Stream North, Stream South, and Off-Stream South, respectively.

The calibration of the absolute magnitude of the TRGB was intensively studied by Jang \& Lee (2017), and various calibrations are listed in Jang \& Lee (2017). For the absolute magnitude of the TRGB, we adopt the calibration by Rizzi et al. (2007):

$$
\begin{aligned}
M_{I_{c}}(\mathrm{TRGB})= & -4.05( \pm 0.02)+0.22( \pm 0.01) \\
& \times\left[\left(V-I_{c}\right)-1.6\right] .
\end{aligned}
$$


Table 2

Estimated TRGB Magnitude for the Four Regions

\begin{tabular}{lccccccc}
\hline \hline Region & Mean & Peak & $5 \%$ & $25 \%$ & $50 \%$ & $75 \%$ & $95 \%$ \\
\hline $\begin{array}{l}\text { Stream } \\
\quad \text { North }\end{array}$ & 20.90 & 20.90 & 20.87 & 20.89 & 20.90 & 20.91 & 20.92 \\
$\begin{array}{l}\text { Stream } \\
\quad \text { South }\end{array}$ & 20.87 & 20.87 & 20.85 & 20.86 & 20.87 & 20.87 & 20.89 \\
$\begin{array}{l}\text { Off-Stream } \\
\quad \text { South }\end{array}$ & 20.79 & 20.84 & 20.58 & 20.78 & 20.83 & 20.85 & 20.86 \\
$\quad$ & & & & & & \\
\hline
\end{tabular}

When we use the color conversion formula for the relevant range of $1.3<g-i<1.7$ (see the Appendix),

$$
\begin{aligned}
& V-I_{c}=0.715(g-i)+0.317, \\
& i-I_{c}=0.067(g-i)+0.426,
\end{aligned}
$$

we obtain

$$
M_{i}(\mathrm{TRGB})=0.222(g-i)-3.902 .
$$

For the TRGB of Stream North, we find $i_{0}=20.90 \pm 0.033$ and $(g-i)_{0}=1.7$, where $(g-i)_{0}$ is the mean color of the TRGB, and thus Equation (6) yields $(m-M)=24.42$. Similarly, we obtain $(m-M)=24.39$ and $(m-M)=24.31$ for Stream South and Off-Stream South.

The error of $(m-M)$ is calculated as follows: for the random error, we take the errors from the Rizzi equation (Equation (3)), the error from the TRGB determination $(\sigma=0.02)$, and the width of the TRGB color $(\sigma=0.1)$, and then calculate the root square sum of all propagated errors. For the systematic error, we take the errors from the fitting of the color conversions ( $\Delta=0.017$ and $\Delta=0.009$ for Equations (4) and (5)) and the absolute photometric calibration $(\Delta=0.02)$ and then calculate the sum of all propagated errors. The random and systematic errors are calculated to be 0.033 and 0.033 , respectively, for Stream North and South, and those for Off-Stream South are 0.040 and 0.033 . The distances derived from the TRGBs and their errors are summarized in Table 3.

\subsection{Stellar Population of the RGB, RC, and RGB Bump}

To determine the absolute luminosities of specific features in a CMD, such as the RC and RGB bump (RGBb), we need to set the age, $\tau_{\text {age }}$, and metallicity, $Z$, of the stellar population in both stream and off-stream regions. For this purpose, we attempt to reproduce both the locus of the RGB and the relative position of the RC in a CMD for the ranges of $\tau_{\text {age }}$ and $Z$ based on a Padova isochrone with a fiducial value for the mass-loss parameter on the RGB of $\eta=0.2-0.5$. The color system of the isochrone (SDSS system) is converted to the HSC system as described in the Appendix.

First, we adopt the distance modulus determined using the TRGB method described in Section 4.1 and look for the best-fit isochrone. For Off-Stream South, we find that the isochrone with $Z=0.0014([\mathrm{Fe} / \mathrm{H}]=-1.13), \log \tau_{\text {age }}(\mathrm{Gyr})=10.00$, and $\eta=0.3$ traces the RGB and RC features perfectly (see Figures 8(4b) and (4c)). On the other hand, we found it difficult to fit the isochrones to the CMDs of Stream North and Stream South, in particular to the RC features (see thick black lines in Figures 8(1c) and (3c)). We therefore try to fit the isochrone by changing the distance modulus and find that the isochrone with $Z=0.0008([\mathrm{Fe} / \mathrm{H}]=-1.37), \log \tau_{\text {age }}(\mathrm{Gyr})=10.00$, and $\eta=0.3$ traces the RGB and RC features for Stream North and

\begin{tabular}{|c|c|c|c|}
\hline Field & Stream North & Stream South & Off-Stream South \\
\hline $\begin{array}{l}(m-M) \text { from TRGB } \\
\text { (random error) } \\
\text { (systematic error) }\end{array}$ & $\begin{array}{l}24.42 \\
\pm 0.033 \\
\pm 0.033\end{array}$ & $\begin{array}{l}24.39 \\
\pm 0.033 \\
\pm 0.033\end{array}$ & $\begin{array}{l}24.36 \\
\pm 0.040 \\
\pm 0.033\end{array}$ \\
\hline $\begin{array}{l}(m-M) \text { from } \mathrm{RC} \\
\text { (random error) } \\
\text { (systematic error) }\end{array}$ & $\begin{array}{l}24.63 \\
\pm 0.191 \\
\pm 0.057\end{array}$ & $\begin{array}{l}\cdots \\
\cdots \\
\cdots\end{array}$ & $\begin{array}{l}24.29 \\
\pm 0.211 \\
\pm 0.057\end{array}$ \\
\hline$(m-M)$ from $\mathrm{RGBb}$ & 24.77 & $\cdots$ & 24.39 \\
\hline
\end{tabular}

Table 3

Summary of the Distances Calculated in This Study

Stream South by increasing the distance modulus by 0.3 and $0.2 \mathrm{mag}$, respectively (see black dashed lines in Figures 8(1c) and (3c)). We note that the "deep dip" in the LF is found for Stream North at $i_{0} \simeq 21.14 \mathrm{mag}$, which is in contrast to the monotonic increase of the RGB population toward fainter magnitudes found for most galaxies. This may suggest that the real RGB population in Stream North emerges from $i_{0} \sim$ $21.14 \mathrm{mag}$. The edge-detection filter suggests the peak to be at $i_{0} \simeq 21.22$, which is consistent with the suggestion from isochrone fitting. Note that the typical metallicity range estimated from the width of the RGB is $\sigma_{[\mathrm{Fe} / \mathrm{H}]} \sim 0.2$, which is used in what follows.

\subsubsection{Red Clump}

Figure 10 shows the zoomed-in view of the CMDs in the form of a density map around the $\mathrm{RC}$, where we note that the vertical axis is now the $g$-band magnitude in this figure. Significant peaks of the RC at $(g-i)_{0} \simeq 0.6$ are found in the CMDs for the Stream North, Stream South and the Off-Stream South regions. It is also suggested that the CMD around the RC for Stream South can be reproduced by the combination of the CMDs for Stream North and Off-Stream South. No significant peak is found for Off-Stream North, but this is naturally understood if Off-Stream North region is a representative of a smooth halo (see Section 3.2).

We measure the color and magnitude of the peak of the $\mathrm{RC}$ for each region. They are measured to be $(g-i)_{0}=0.562 \pm 0.085$ and $g_{0}=25.456 \pm 0.132$ for Stream North and $(g-i)_{0}=$ $0.568 \pm 0.080$ and $g_{0}=25.122 \pm 0.160$ for Off-Stream South. Here, we adopt the standard deviation around the peak of the RC as errors. Since two different structures are suggested to be overlapping in the Stream South region and no significant peak is found for Off-Stream North, we do not attempt to derive the color and magnitude of the RC for these two regions.

For the absolute magnitude of the $\mathrm{RC}$, we adopt the calibration by Bilir et al. (2013):

$$
\begin{aligned}
M_{V}(\mathrm{RC})= & 0.627( \pm 0.104)(B-V)_{0} \\
& +0.046( \pm 0.043)[\mathrm{Fe} / \mathrm{H}] \\
& +0.262( \pm 0.111) .
\end{aligned}
$$

When we use the color conversion formula for the relevant range of $0.4<g-i<1.2$ (see the Appendix),

$$
\begin{aligned}
& g-V=0.371(g-i)+0.068, \\
& B-V=0.709(g-i)+0.170,
\end{aligned}
$$




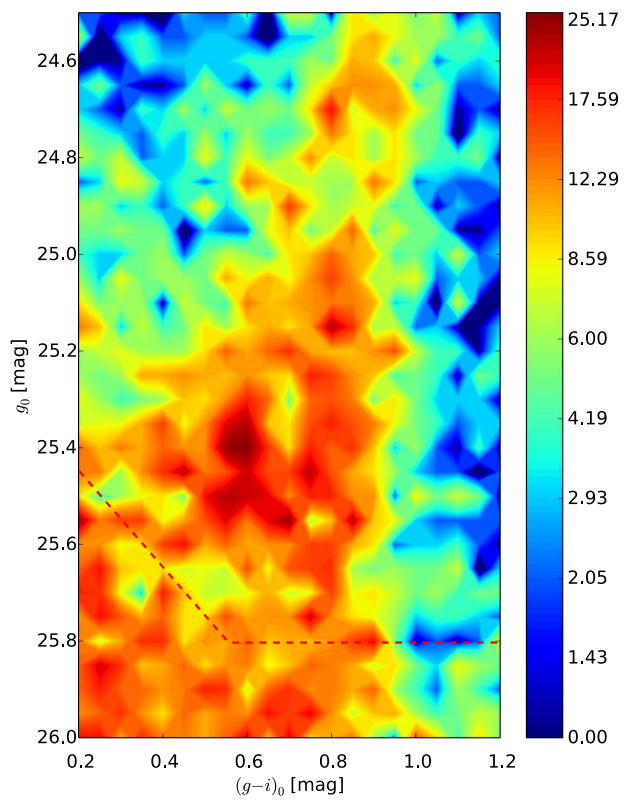

(a) Stream North

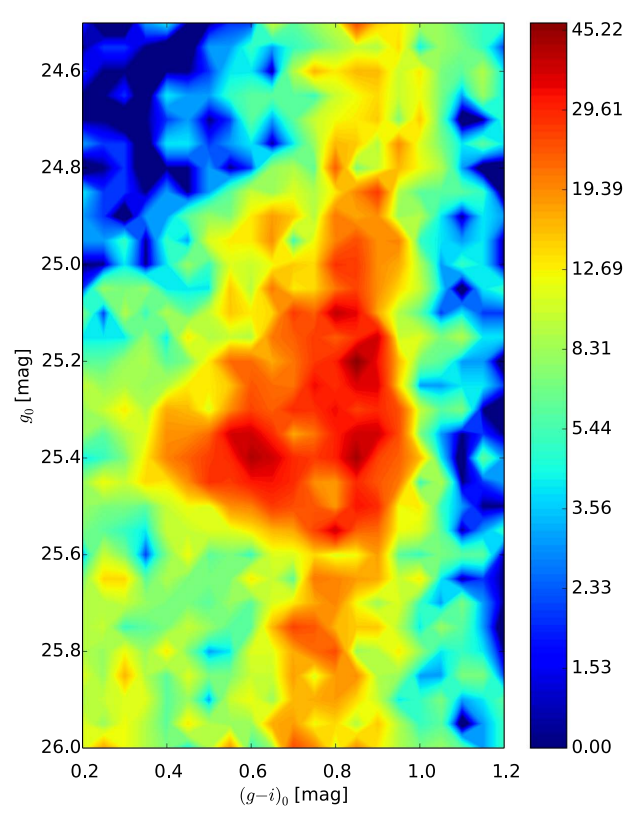

(c) Stream South

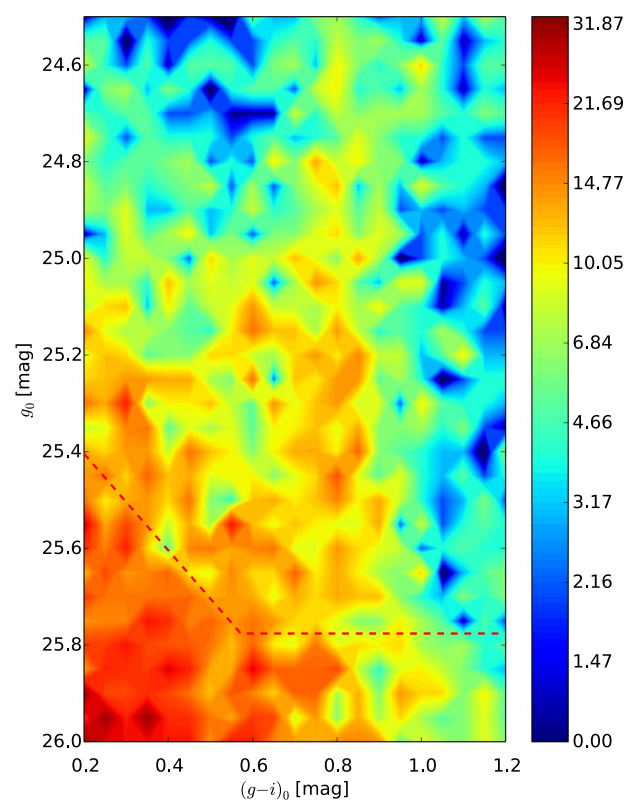

(b) Off-Stream North

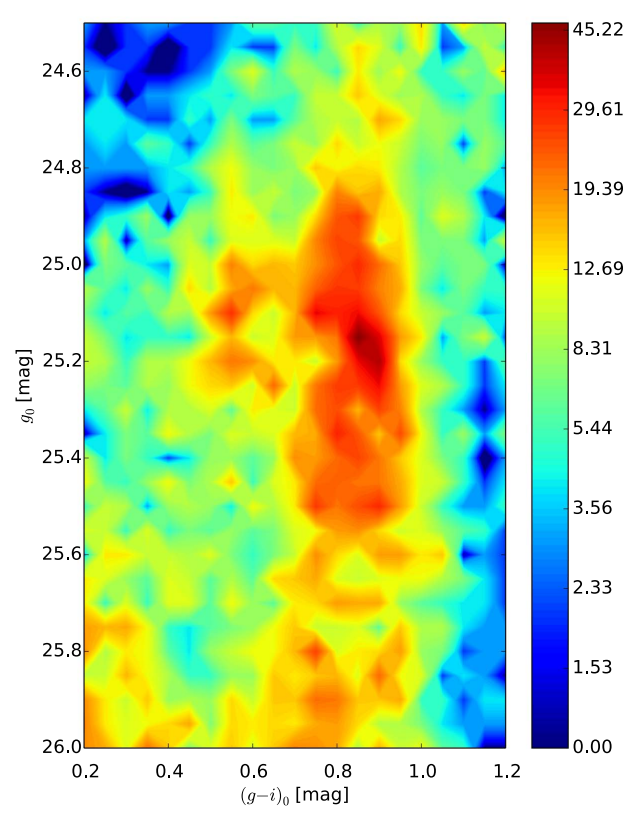

(d) Off-Stream South

Figure 10. Zoomed-in CMDs in the form of a density map around the RC of the four regions in our survey field. From top left to bottom right, the CMDs of (a) Stream North, (b) Off-Stream North, (c) Stream South, and (d) Off-Stream South are plotted. The grid widths used in these figures are $\Delta(g-i)=0.05$ and $\Delta g=0.05$ mag. Note that the vertical axis is the $g$-band magnitude.

we obtain

$$
M_{g}(\mathrm{RC})=0.8155(g-i)+0.046[\mathrm{Fe} / \mathrm{H}]+0.4366 .
$$

Using Equation (10) combined with the metallicity of $[\mathrm{Fe} / \mathrm{H}]=$ -1.38 (see Section 4.2), we obtain $M_{g}(\mathrm{RC})=0.831$ and thus $(m-M)=24.63$ for the RC of Stream North. For the RC ofOffStream South, we obtain $M_{g}(\mathrm{RC})=0.848$ and thus $(m-M)=$ 24.27.

The error of $(m-M)$ is calculated in a similar way to the calculation for the TRGB. For the random error, we take the errors from the Bilir equation (Equation (7)), the error of the RC magnitude, the error of the $\mathrm{RC}$ color, and the error of the metallicity $(\sigma=0.2)$, and then calculate the root square sum of all propagated errors. For the systematic error, we take the errors from the fitting of color conversions $(\Delta=0.029$ and $\Delta=0.019$ for Equations (8) and (9)) and the absolute photometric calibration $(\Delta=0.02)$, and then calculate the sum of all propagated errors. The random and systematic errors are calculated to be $0.191-0.211$ and 0.057 , respectively. The distances derived from the RC and their errors are summarized in Table 3.

\subsubsection{RGB Bump}

In Figure 10, the RGBb features are recognized at $(g-i)_{0} \simeq$ 0.8 for Stream North, Stream South, and Off-Stream South. This 
feature is most prominent at Off-Stream South, suggesting that this feature at $(g-i)_{0} \simeq 0.8$ and $g_{0} \simeq 25.2$ is characteristic for this region, i.e., the diffuse substructure. Looking at the CMD of Stream South, a peak is found at the same position as in Stream South, and the other peak is found at $(g-i)_{0} \simeq 0.8$ and $g_{0} \simeq 25.4$, which is suggested to be the bump feature of the M31 NW Stream and should also be found at Stream North. Bearing this in mind, we measure the color and magnitude of the $\mathrm{RGBb}$ feature for the Stream North and Off-Stream South regions. They are measured to be $(g-i)_{0}=0.830 \pm 0.076$ and $g_{0}=25.478 \pm 0.097$ for Stream North and $(g-i)_{0}=$ $0.857 \pm 0.076$ and $g_{0}=25.112 \pm 0.110$ for Off-Stream South.

Alvez \& Sarajedini (1999) give a table of the $V$-band absolute magnitudes of the RGBb, $M_{V}(\mathrm{RGBb})$, for stellar populations with different ages and metallicities. The interpolation of the table gives $M_{V}(\mathrm{RGBb})=0.177$ and $M_{V}(\mathrm{RGBb})=0.477$ for Stream North and Off-Stream South, respectively. Thus, using Equation (8) to estimate the $V$-band magnitude from the values of $g_{0}$ and $(g-i)_{0}$ of the RGBb, we obtain the distance modulus $(m-M)$ to each region. For the $\mathrm{RGBb}$ of Stream North, our estimate of $(g-i)_{0}=0.830$ and $g_{0}=25.478$ yields $V=25.102$, thus $(m-M)=24.93$. For the $\mathrm{RGBb}$ of Off-Stream South, our estimate of $(g-i)_{0}=0.857$ and $g_{0}=25.112$ yields $V=24.726$, thus $(m-M)=24.25$. The derived distance moduli are consistent with the tendency obtained from the RC methods that Stream North (the NW Stream) is located at farther than Off-Stream South (the diffuse substructure). Note that the Alvez \& Sarajedini (1999) relation seems to be very sensitive to the metallicity and age of the population, and the present estimate would have a large uncertainty.

\subsection{Summary of Distance Estimates of Stellar Populations}

Table 3 summarizes the distance moduli measured using the different methods presented here. The Off-Stream South region shows a small variation in the distance moduli estimated with the different methods. However, the difference between the distance modulus derived from the TRGB and that from the RC is large for the Stream North region. This difference is relaxed if the distance modulus from the TRGB is increased by $0.2-0.3 \mathrm{mag}$, which is suggested from the isochrone fitting (see Section 4.2). We therefore prefer to use the distance moduli derived from RC method for the following analysis.

Considering that the distance modulus of M31 is $24.45 \mathrm{mag}$ (776 kpc), which is the median of the 345 measurements compiled in the NASA/IPAC Extragalactic Database (NED), ${ }^{22}$ the NW Stream of our survey field is located $\sim 90 \mathrm{kpc}$ behind M31 if we adopt the distance modulus derived from the RC for Stream North. By contrast, the diffuse substructure, represented by Off-Stream South, found in this study is located more than $\sim 30 \mathrm{kpc}$ in front of M31.

\subsection{D Structure of the M31 NW Stream}

To understand the nature of the M31 NW Stream, it is important to obtain the distance information for the M31 NW Stream. If the 3D structure of the NW Stream is obtained, then this information gives a useful constraint on the origin of the stream.

\footnotetext{
${ }^{22}$ http://ned.ipac.caltech.edu/
}

In this section, we investigate the distances to the NW Stream by dividing the stream regions (i.e., Stream North and South) into four regions. The boundaries are set as $\delta=43.7,44.8$, and 46.1, and we name the regions Stream 1 to 4 from north to south. Due to the issues with the TRGB distances discussed in the previous section, we adopt the RC method as described in Section 4.2.1 to address the distance distribution along the NW stream.

Figure 11 shows the background-/foreground-subtracted CMDs (HESS diagrams) around the RC. The CMD of the background/foreground objects for the Stream 1 region is made from those stars that reside outside the stream region with the same declination range as the Stream 1 region (i.e., $\delta>46.1$ ). The CMDs of the background/foreground objects for the Stream 2-4 regions are made in the same way. In each panel, individual stars in the stream regions are also plotted as black dots, as we aim to show the real distribution of stars in each CMD. We measure the mean color and magnitude of the $\mathrm{RC}$ for each region and find $(g-i)_{0}=0.562 \pm 0.085$ and $g_{0}=25.467 \pm 0.139,(g-i)_{0}=0.562 \pm 0.084$ and $g_{0}=25.447 \pm 0.125,(g-i)_{0}=0.569 \pm 0.085$ and $g_{0}=$ $25.430 \pm 0.119$, and $(g-i)_{0}=0.573 \pm 0.083$ and $g_{0}=$ $25.420 \pm 0.123$ for the Stream 1-4 regions, respectively. The distance moduli derived from the RC method are 24.64, 24.62, 24.59, and 24.58 for Stream 1 to 4 , respectively (see Table 4). Note that Stream 1 is shallow in terms of the completeness, but the peak of the RC seems to be reasonably measured since it is found to be $\sim 0.2$ mag brighter than the $50 \%$ completeness limit.

As explained in Section 2.2, the Galactic extinction is corrected for every single star using the new estimate from Schlafly \& Finkbeiner (2011), which is based on the dust map by Schlegel et al. (1998). The reddening $E(B-V)$ ranges from $\sim 0$ to 0.1318 within our survey field (see Figure 4 ), which corresponds to the maximum extinction of $A_{g}=0.4858$, $A_{i}=0.2440$. Therefore, the error in the reddening map would introduce an additional error to the extinction-corrected magnitudes. It is noted that the variation in the extinctioncorrected color $(g-i)_{0}$ of the RC along the stream is as small as 0.01 mag. This suggests that our method to correct extinction is carried out properly given that the stellar population is homogeneous along the stream.

Although the uncertainty in the RC method is large, the result indicates that the southern part of the NW Stream is $\sim 20 \mathrm{kpc}$ closer to us (and M31) relative to the northern part (see the right panel of Figure 16). The angle between Stream 1 and 4 is $\sim 4.2$, which corresponds to the projected distance of $\sim 61 \mathrm{kpc}$, indicating that this configuration seems to be realistic. The angle from Stream 4 to the major axis of M31 along the stream is roughly $6^{\circ}$ (see Figure 1), which corresponds to the projected distance of $\sim 90 \mathrm{kpc}$. The line-of-sight distance between Stream 4 and M31 is calculated to be $\sim 50 \mathrm{kpc}$. If the NW Stream is extended to the southeast direction with the same rate as that found in our survey field, it is still behind M31 when it crosses the major axis of M31. Therefore, we conclude that the NW Stream is part of an orbiting stream around the farther side of M31.

\subsection{Shape of the M31 NW Stream}

Figure 12 shows the number density distributions of RC stars for the Stream 1-4 fields along the direction perpendicular to the NW Stream, where the coordinate Xr increases toward the 


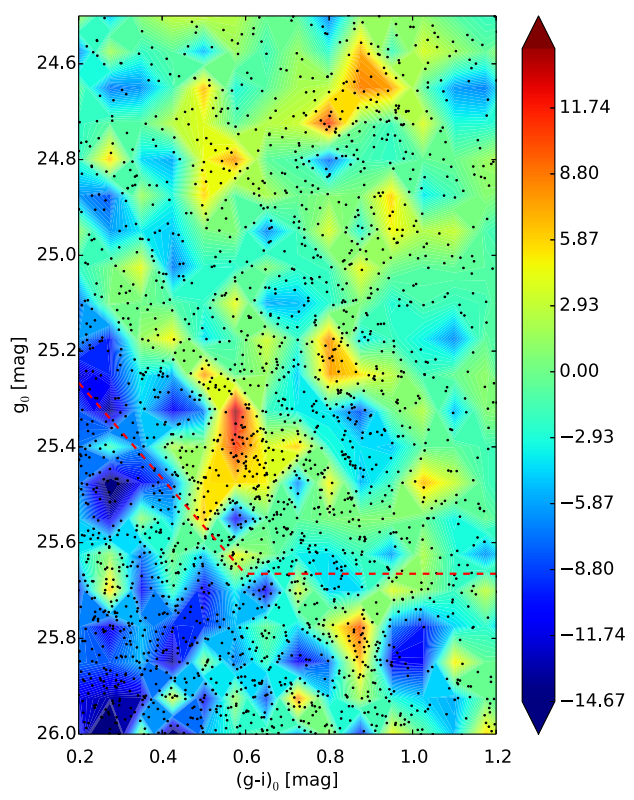

(a) Stream 1

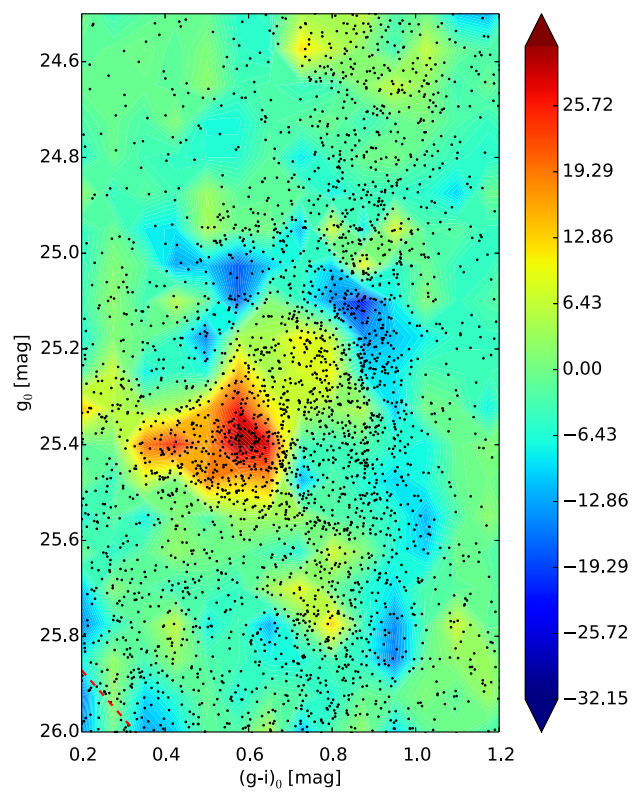

(c) Stream 3

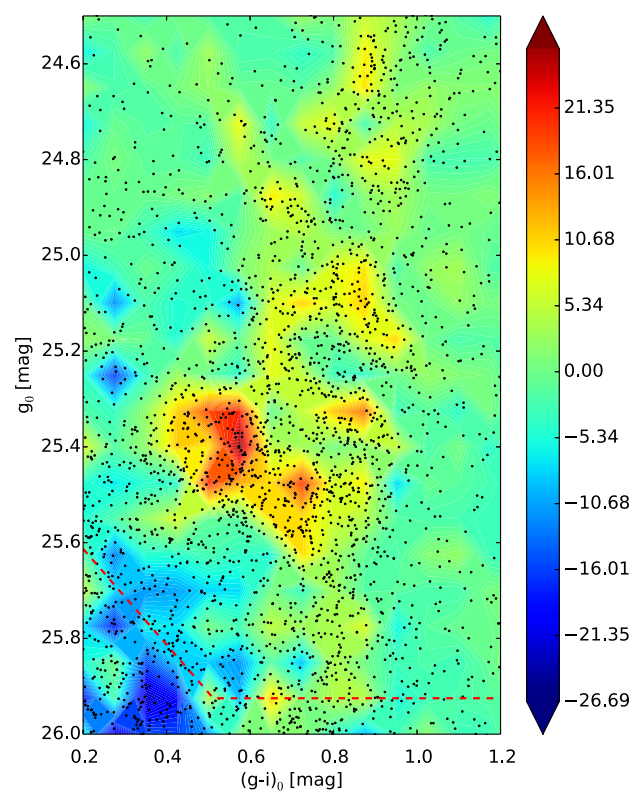

(b) Stream 2

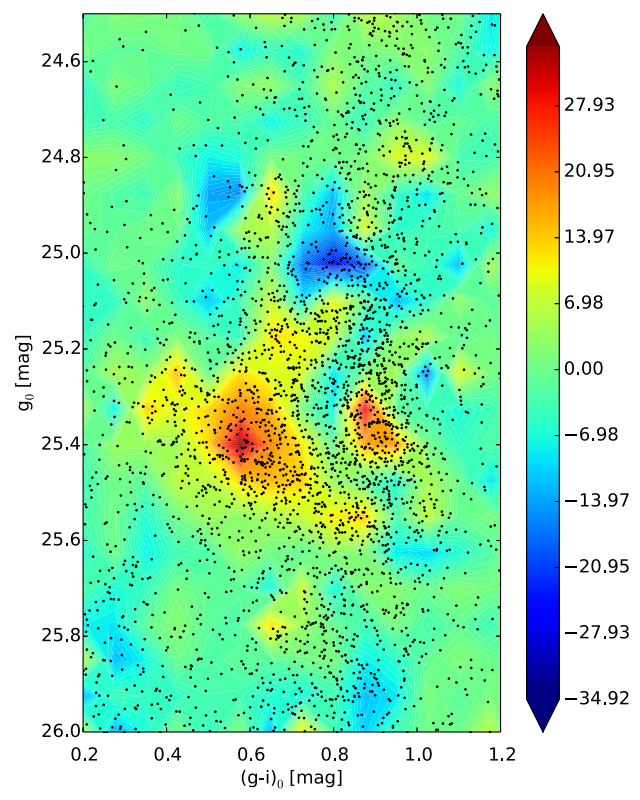

(d) Stream 4

Figure 11. Background-/foreground-subtracted CMDs (HESS diagram) around the RC of the four regions along the NW Stream. From top left to bottom right, the CMDs of (a) Stream 1, (b) Stream 2, (c) Stream 3, and (d) Stream 4, respectively, are plotted. In each panel, individual stars in the stream region are also plotted as black dots. The grid widths used in these figures are $\Delta(g-i)=0.075$ and $\Delta g=0.075 \mathrm{mag}$.

Table 4

Summary of Distances of the NW Stream

\begin{tabular}{lcccc}
\hline \hline Field & $\begin{array}{c}\text { Center Coordi- } \\
\text { nates (degree) }\end{array}$ & $(m-M)$ & $\begin{array}{c}(m-M) \text { Error (ran- } \\
\text { dom/systematic })\end{array}$ & $\begin{array}{c}\text { Distance } \\
(\mathrm{kpc})\end{array}$ \\
\hline Stream 1 & $(2.06,46.90)$ & 24.64 & $\pm 0.196 \pm 0.057$ & 847 \\
Stream 2 & $(3.00,45.45)$ & 24.62 & $\pm 0.186 \pm 0.057$ & 839 \\
Stream 3 & $(3.78,44.25)$ & 24.59 & $\pm 0.183 \pm 0.057$ & 828 \\
Stream 4 & $(4.52,43.10)$ & 24.58 & $\pm 0.185 \pm 0.057$ & 824 \\
\hline
\end{tabular}

southwest direction as shown in the top panel of Figure 13. Although our survey fully covers neither the southwest side (i.e., $\mathrm{Xr}>18$ ) of the Stream 1 field nor the northeast side (i.e., $\mathrm{Xr}<-30 \sim-24)$ of the Stream 2-4 fields, the figures for Stream 2 and 3 clearly show the number density distribution across the NW Stream.

The overall shape can be fitted by the combination of a Gaussian with an FWHM of $\sim 25$ arcmin and a linear function for the Stream 2 and 3 fields. It is noted that the distribution seems to be slightly skewed: the southwest side of the stream (i.e., the right side of the distribution in the figures) shows a steep rise $\left(\sim 0.06 \operatorname{arcmin}^{-3}\right.$ for $\left.6<\mathrm{Xr}<18\right)$ while the northeast side shows a rather shallow rise $\left(\sim 0.03 \mathrm{arcmin}^{-3}\right.$ for $-18<\mathrm{Xr}<-6$ ) in the Stream 3 field. A similar trend is 


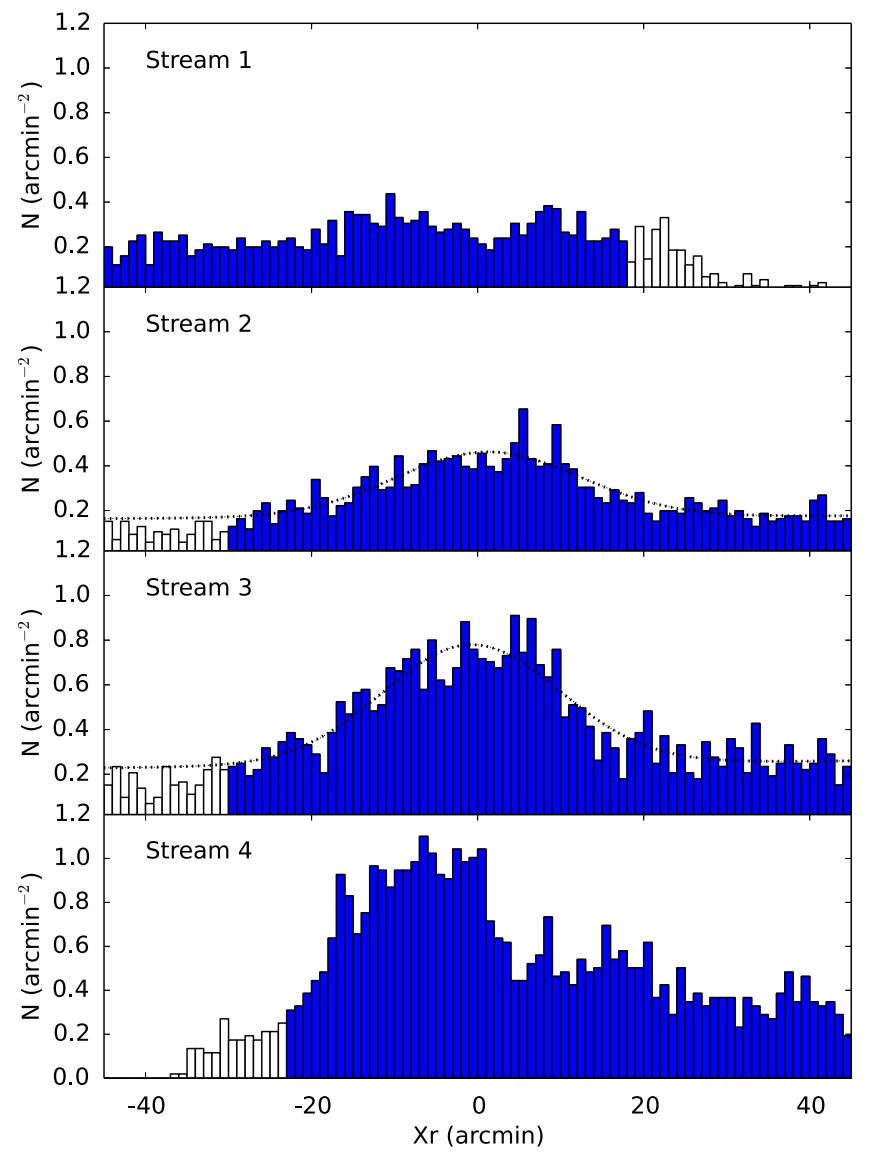

Figure 12. Number density distributions of RC stars along the direction perpendicular to the NW Stream. From top to bottom, the histograms for Stream 1-4 are shown. Positive Xr corresponds to the southwest side of the NW Stream. The areas shown by the white histograms are where our survey coverage is incomplete. The best-fit profile, which is a combination of a Gaussian and a linear function, is plotted as a dotted line in the Stream 2 and 3 fields.

also seen for the Stream 2 and 4 fields. This may tell us something about the formation process of the NW Stream. The Stream 1 field shows no prominent peak at around $\mathrm{Xr}=0$, but a hint of an overdensity is clearly visible from Figure 7 . The overdensity seems to be extended to the northwest, beyond our survey fields, and thereby further observation in this direction is required to investigate the detailed shape of the Stream 1 field.

The middle panel of Figure 13(a) shows the number density distributions of the RC stars along the NW Stream, where the coordinate $\mathrm{Yr}$ increases toward the northwest direction. The blue histogram represents the surface number density of RC stars within a width of 44.8 arcmin centered on the stream. The width of 44.8 arcmin corresponds roughly to $\pm 2 \sigma$ of the Gaussian fitted for Stream 2 and 3. The positive Yr corresponds to the northwest side of the NW Stream as shown in the top panel of Figure 13(a). The green and red histograms represent those for outside the stream on the north and south sides, respectively. The histograms are calculated using those $\mathrm{RC}$ stars found in the $1.5 \sigma$ width regions, although our survey does not always cover these regions. Therefore, the red and green histograms should be taken as a guide to estimate the foreground/background contamination of the surface number density of RC stars of the NW Stream.
The blue histogram outnumbers the green and red histograms for most of the figure, indicating that the stream is found all throughout our survey fields. We also note that the surface number density varies dramatically along the stream, and several gaps are found in the stream. We try to estimate the foreground-/ background-subtracted number density distribution of RC stars assuming that the maximum of the green and red histograms at fixed Yr represents the foreground/background. The bottom panel of Figure 13(a) shows the foreground-/background-subtracted number density distributions in each bin (dotted line) and a moving average over five bins (thick line). The surface number density is highest at $\mathrm{Yr}=13 \mathrm{arcmin}$ and is $0.66 \pm 0.10 \mathrm{arcmin}^{-2}$ (Poisson error is assumed). The overall surface number density gradually decreases as Yr increases, with bumps and dips along the stream. Since the typical error ranges from 0.05 to $0.1 \operatorname{arcmin}^{-2}$, most of the bumps are statistically significant. The most significant gap is found at $210<\mathrm{Yr}<240$, which is also clearly seen in Figure 7. It is again recovered at $\mathrm{Yr}>240$, although the surface number density is the lowest among our survey fields. However, the significance of this rise is uncertain since completeness lower than $50 \%$ for $\mathrm{Yr}>240$ is suggested from Figure 7(b). Comparing the top and bottom panels of Figure 13(a), the peak positions are not coincident with the globular clusters found in the NW Stream (Huxor et al. 2014).

We also made a similar figure for NBGs as shown in Figure 13(b). Since the error in the surface number density is calculated to be between 0.02 and $0.05 \operatorname{arcmin}^{-2}$ (Poisson error is assumed), the significance of the features seen in the bottom panel of Figure 13(b) is low compared to that of Figure 13(a). But it is suggested that the overall shape along the stream, such as the gap at $210<\mathrm{Yr}<240$, the bump at $150<\mathrm{Yr}<200$, and the gradual increase in the number density distribution toward smaller Yr, is similar between RCs and NGBs except for $\mathrm{Yr}<30$, where the derived number density seems to be affected by a clump of NBGs found at $(\mathrm{Xr}, \mathrm{Yr})=(30,15)$. Unfortunately, the fine structures, such as the sharp peaks seen in the bottom panel of Figure 13(a), are not confirmed by NBGs due to the shortage of statistical significance.

Recently, many studies on the formation of gaps in stellar streams due to their encounter with $\Lambda \mathrm{CDM}$ substructures have been carried out (e.g., Carlberg et al. 2011; Carlberg 2012; Erkal et al. 2016). Our data provide an excellent observational example to test these simulations. It is also noted that the bumps found in the bottom panel of Figure 13(a) seem to be periodic, which is reminiscent of the epicyclic bumps seen in simulations of star clusters (e.g., Küpper et al. 2012).

Carlberg (2012) investigated the density profile of the NW Stream using PAndAS data (Carlberg et al. 2011). They showed the density profile with a moving average over 5 or 11 bins of $0^{\circ} .25$. This indicates that the resolution of their analysis is $>0.25$, an order lower than ours. Therefore, our data are complementary to theirs and would be of help in understanding the detail of the density profile at the small scale.

\section{Discussion}

\subsection{Properties of Globular Clusters in the NW Stream}

Huxor et al. (2014) reported their discovery of 59 globular clusters (GCs) and two candidates in the halo of M31 based on the PAndAS survey data. Follow-up spectroscopic observations carried out by Veljanoski et al. (2014) obtained radial 


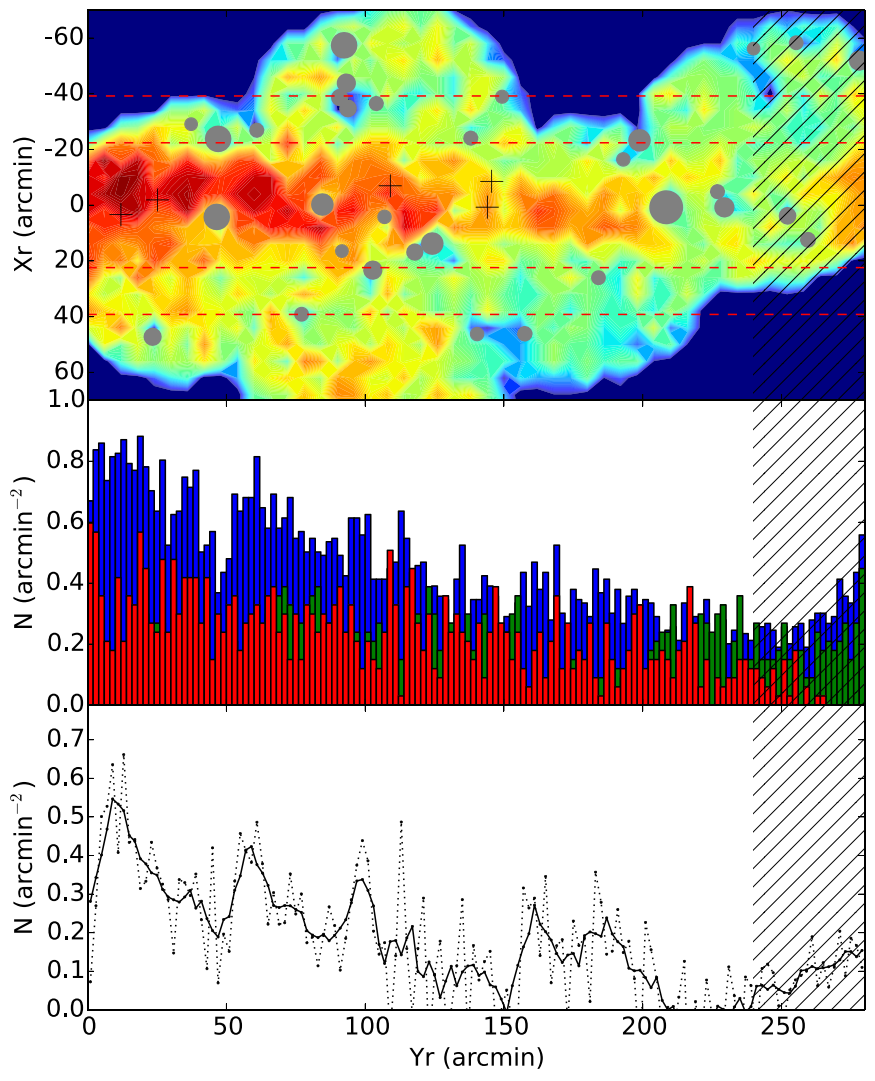

(a)

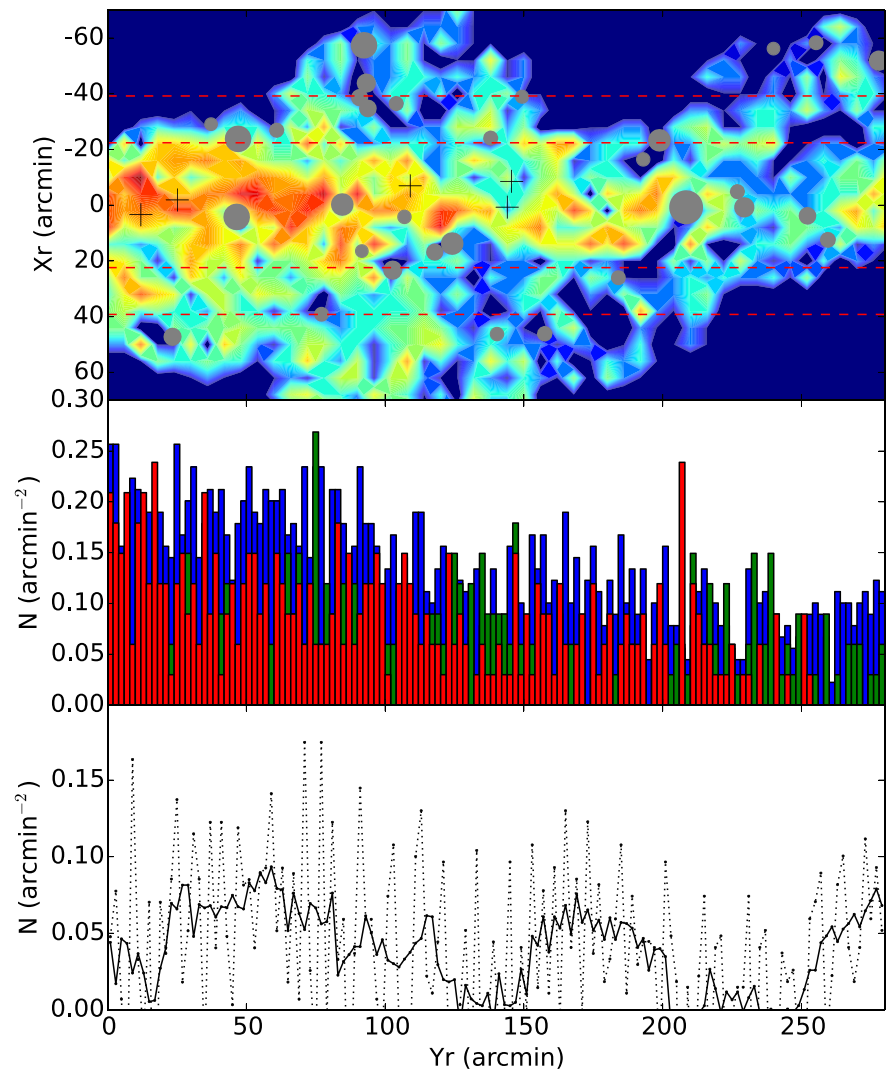

(b)

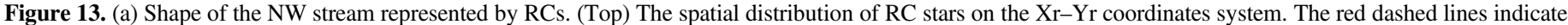

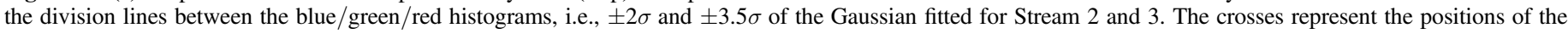

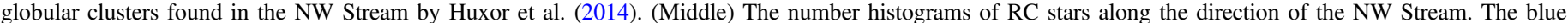

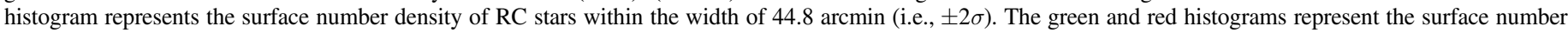

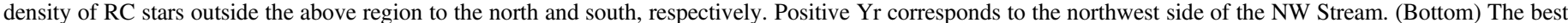

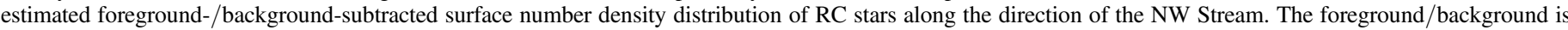

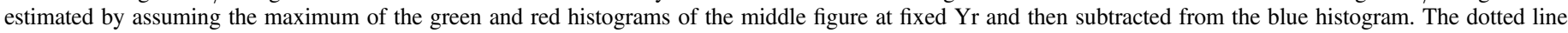

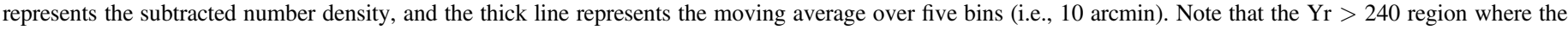
completeness is lower than $50 \%$ as suggested in Figure 7 (b) is hatched in the figure. (b) Same figure as (a), but for NBGs.

velocities for $78 \mathrm{GCs}$. Now, a large sample of GCs is available, and detailed studies of GCs can be possible even for M31.

Among these GCs, seven GCs show a hint of association with the NW Stream, and five share a clear trend in corrected radial velocity as a function of projected radius (Veljanoski et al. 2014), suggesting the same origin as the NW Stream. Our survey field contains five GCs, and they are found to be located near the center of the stream, suggesting their association with the stream (Figure 7). Out of the five GCs, three GCs located at the south of our survey field (PAndAS-11 to 13) clearly trace the high surface number density regions as shown in Figure 7. On the other hand, the remaining two (PAndAS-9 and 10) are located at a relatively low surface number density region $(\mathrm{Yr}=140$ in Figure 13).

Making use of our high image quality data, we carry out a detailed study of these GCs in combination with the NW Stream to investigate the relation between these GCs and the NW Stream. Unfortunately, one GC (PAndAS-9) is buried in the halo of a bright star $(V=10)$, making it difficult to detect stars belonging to the GC. We carried out a detailed analysis for the remaining four GCs, PAndAS-10 to 13. DAOphot PSF photometry software (Stetson 1987, 1994) is used for these GCs, and CMDs based on DAOphot photometry are plotted in Figure 14. Those stars with good fitting quality (Sharpness $<1$ ) are plotted as large dots and others as small dots. The mean photometric errors are calculated for every 1 mag range and plotted as crosses at $(g-i)_{0}=-0.25$. All of the CMDs show clear sequences of RGB stars, although the scatters are slightly larger than that observed for Stream North (Figure 8(a)). In each $\mathrm{CMD}$, an isochrone of the RGB that best traces the distribution of RGB stars $\left(\left(\log \tau_{\text {age }}, Z\right)=(10.12,0.002),(10.00,0.001)\right.$, $(10.00,0.002)$, and $(10.12,0.001)$ for PAndAS-10 to 13 , respectively, with $(m-M)=24.63)$ is plotted for guidance.

Comparing these CMDs with that for Stream North, no significant difference in the position of the RGB in the CMD is seen between Stream North and all of the GCs, indicating that the stellar populations consisting of GCs are similar to that of the stream. It is also noted that the brightest RGB star in each $\mathrm{GC}$ is equal to or fainter than the tip of the isochrone, which is consistent with the condition that GCs are located nearer or farther than the distance of $(m-M)=24.63$, except for PAndAS-13, in which a star with $i_{0}=20.4$ is found. Note that Veljanoski et al. (2014) pointed out that PAndAS-13 is displaced from the linear relation of the radial velocity with the projected radius observed for the other five GCs (PAndAS4, 9-12). The results support the idea that both GCs (PAndAS$10-12)$ and the stream originated from the same progenitor system and share the same orbit in the halo of M31. 


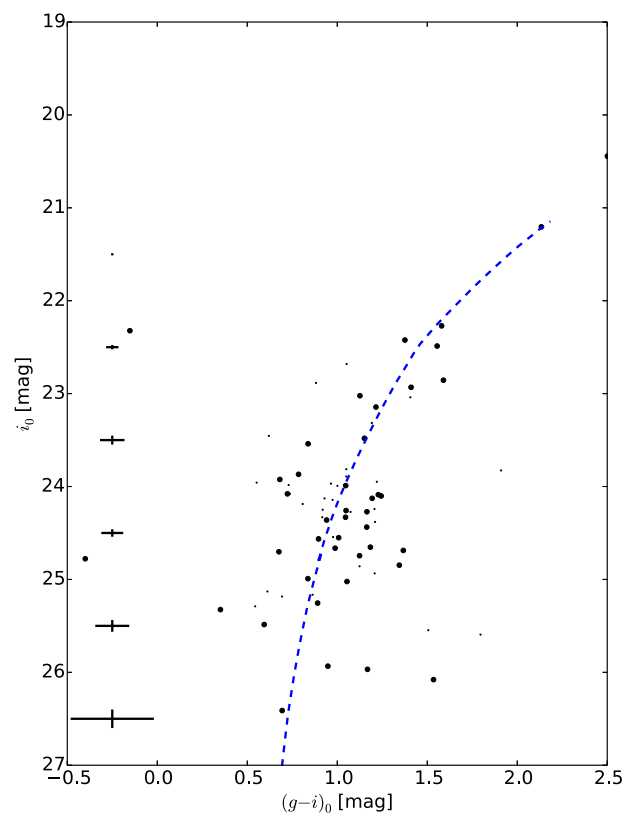

(a) PAndAS-10

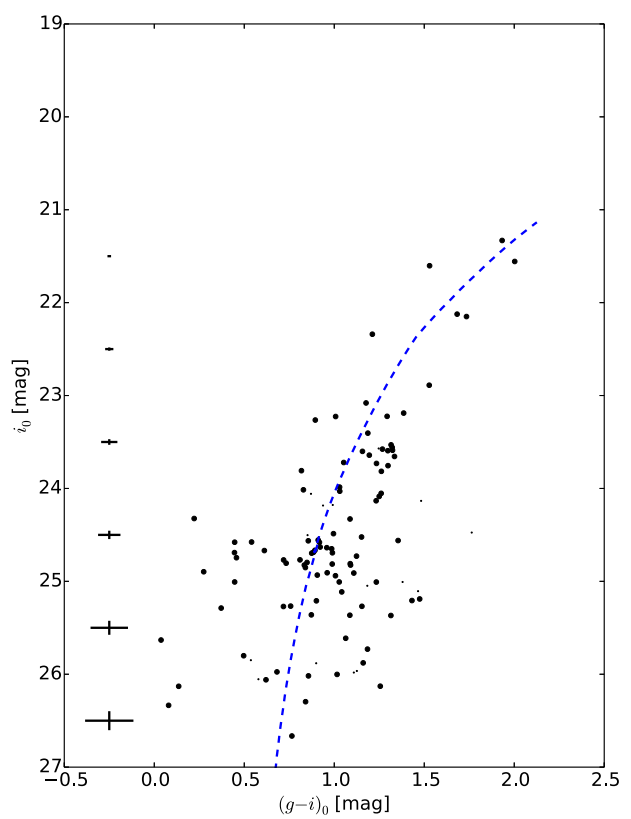

(c) PAndAS-12

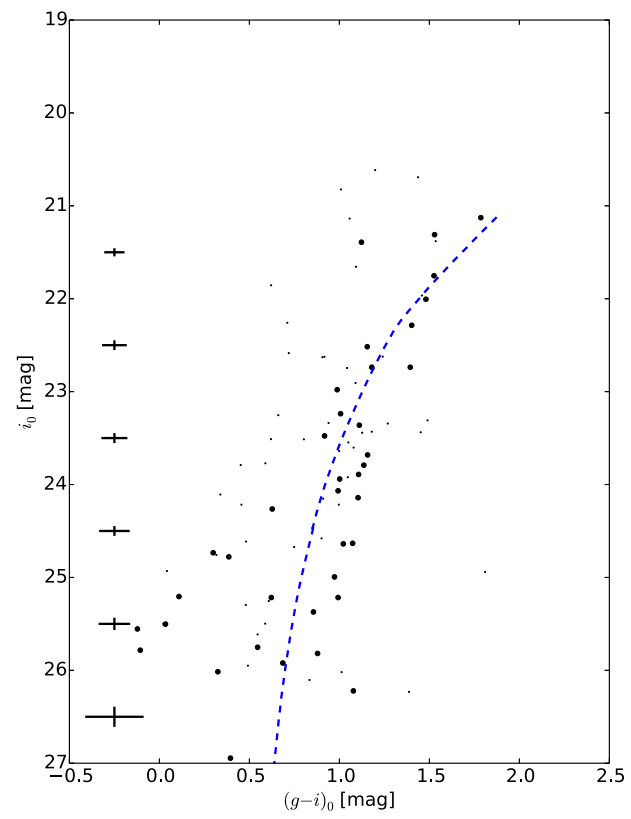

(b) PAndAS-11

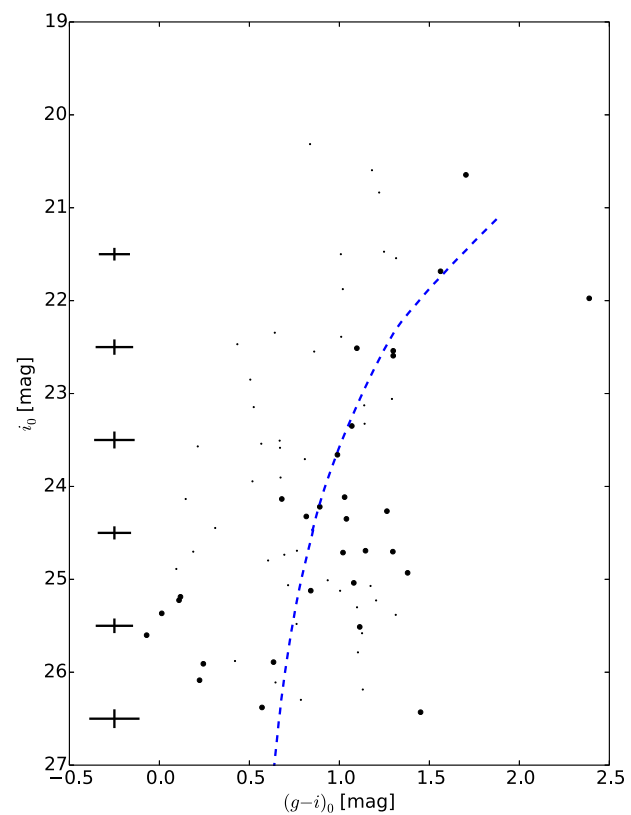

(d) PAndAS-13

Figure 14. CMDs of GCs found on the NW Stream: (a) PAndAS-10, (b) PAndAS-11, (c) PAndAS-12, and (d) PAndAS-13. Large and small dots represent those stars with Sharpness $<1$ and $>1$, respectively. The mean errors are calculated for every 1 mag and plotted as black crosses. An isochrone of the RGB part with (log $\tau_{\text {age }}$, $Z)=(10.12,0.002),(10.00,0.001),(10.00,0.002)$, and $(10.12,0.001)$ is overlaid for PAndAS-10 to 13 , respectively. The distance modulus of $(m-M)=24.63$ is adopted.

We also point out that a small difference in the stellar population of GCs is suggested by the isochrone fitting. This difference is supported by the integrated photometry for these GCs by Huxor et al. (2014), which shows that PAndAS-10 and 12 are redder $\left((g-i)_{0}=0.75\right.$ and 0.75 , respectively) than PAndAS-11 and $13\left((g-i)_{0}=0.67\right.$ and 0.65 , respectively). Therefore, the small difference in the stellar population of GCs seems to be real. Follow-up spectroscopic observations to measure the metallicity would give an answer regarding the stellar population of these GCs.

\subsection{Comparison with Simulation}

Kirihara et al. (2017a) recently carried out an extensive numerical simulation to constrain the orbits and physical properties of the progenitor of the NW Stream, provided that the five GCs in the NW Stream (PAndAS-04, 09, 10, 11 and 12) share the same orbit as the NW Stream, thereby making use of the radial velocities of these GCs. They showed that the allowable orbits of the NW Stream are divided into two branches: one wherein the stream is located in front of M31 with line-of-sight distances of the apocenter $D_{\text {apo }}$ of $430-680 \mathrm{kpc}$, 

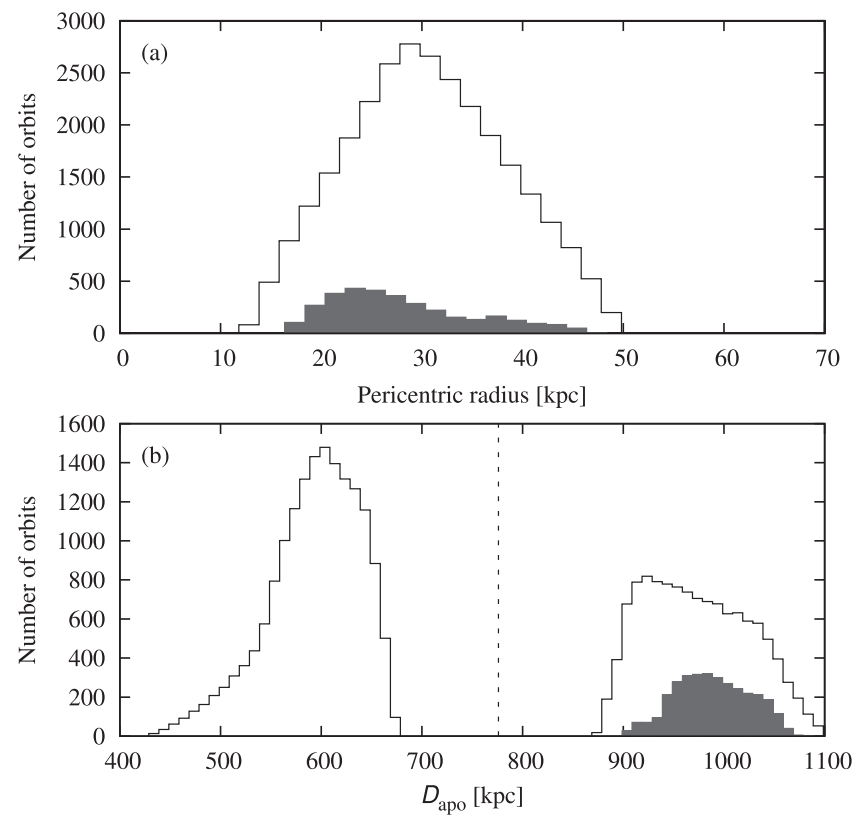

Figure 15. Histogram of (a) the pericentric radii and (b) the apocenter $D_{\text {apo }}$ of the potential orbits. The thin lines indicate the possible orbits basically derived by Kirihara et al. (2017a). The shaded area corresponds to the new orbits derived in this work, including the observed distance to the NW Stream. The vertical dashed line in panel (c) marks the distance to M31 from the Earth.

and the other wherein the stream is located behind M31 with $D_{\text {apo }}$ of 880-1100 kpc (see also Figure 15(b)).

The key to constrain the orbit is the line-of-sight distance to the NW Stream, which we derived to be $824-847 \mathrm{kpc}$ from the RC method (see Table 4). Combined with our distance measurement, we carried out test-particle simulations. A test particle is launched from the position of the GC PAndAS-12 in the gravitational potential of M31. The potential consists of a Hernquist bulge (Hernquist 1990), an exponential disk, and an NFW dark matter halo (Navarro et al. 1998) following Kirihara et al. (2017b). The M31 bulge is set to have the scale radius of $0.61 \mathrm{kpc}$ and the total mass of $3.24 \times 10^{10} M_{\odot}$. The scale height, radial scale length, total mass, and central surface density of the M31 disk are $0.6 \mathrm{kpc}, 5.4 \mathrm{kpc}, 3.66 \times 10^{10} M_{\odot}$, and $2.0 \times 10^{8} M_{\odot} \mathrm{kpc}^{-2}$, respectively. The inclination and position angle of $\mathrm{M} 31^{\prime}$ 's disk are $77^{\circ}$ and $37^{\circ}$, respectively (Geehan et al. 2006). The scale radius and scale density of the NFW halo are $7.63 \mathrm{kpc}$ and $6.17 \times 10^{7} M_{\odot} \mathrm{kpc}^{-3}$, respectively. The radial velocity of the test particle is set to $472 \mathrm{~km} \mathrm{~s}^{-1}$, which is the observed value of PAndAS-12 (Veljanoski et al. 2014). Its initial distance and proper motion velocities are systematically varied following Kirihara et al. (2017a). To find the acceptable ranges of orbital parameters, we conduct a $\chi_{\nu}^{2}$ analysis for the observed position of the NW Stream (see Table 1 of Kirihara et al. 2017a) and radial velocities of the five GCs. Successful orbits satisfy the $1 \sigma$ confidence level for both criteria and have experienced two or more apocentric passages within 12 Gyr. We also use the NW Stream distance obtained from the RC for Stream 1-4. Additional constraints in the testparticle simulations are as follows: (1) the test particle passes within 0.5 from the center of each field and (2) the distance matches the observation within the observed uncertainty. Although the distance data derived with the RC for Stream 1-4 have rather large errors, they give a strong constraint on potential orbits for the progenitor of the NW Stream as shown in Figure 16, and the number of allowable orbits is 3290 in $5,068,617$ orbit models.

The pericentric distance of the progenitor's orbit is one of the great keys to limiting the physical properties of the progenitor. Figure 16(a) shows the number distribution of successful orbits as a function of pericentric radius. The histogram has a peak between a pericentric distance of 20 and $30 \mathrm{kpc}$. It is clear that no orbit can approach M31's center within $17 \mathrm{kpc}$. Even if we adopt the constraint for the $3 \sigma$ confidence level, no orbit can reach $17 \mathrm{kpc}$ from M31's center. This result provides an updated criterion for the physical properties of the progenitor model. Following Kirihara et al. (2017a), we estimate the stripped mass of a progenitor using the Hill radius at the pericenter that defines the tidal radius of the satellite in a gravitational potential of the host system. The half-light radius of the progenitor should be greater than $200 \mathrm{pc}$.

We demonstrate the formation process of the NW Stream using an $\mathrm{N}$-body simulation. We construct the progenitor dwarf galaxy as a Plummer model using the MAGI (Miki \& Umemura 2017). The total mass $M_{\text {tot }}$ and scale radius $r_{\mathrm{s}}$ of the Plummer distribution are set to $M_{\mathrm{tot}}=5 \times 10^{7} M_{\odot}$ and $r_{\mathrm{s}}=1 \mathrm{kpc}$, respectively. The total number of particles is 65,536 , and we use the gravitational octree code GOTHIC (Miki \& Umemura 2017) to run the simulation. We adopt the Plummer softening parameter of $16 \mathrm{pc}$ and the accuracy control parameter of $2^{-7}$. The initial phase-space coordinates of the progenitor for a successful orbit are $\left(\xi, \eta, d, V_{\xi}, V_{\eta}, V_{\mathrm{los}}\right)=$ $(-22.18,-0.60,860.07 \mathrm{kpc},-9.88,-23.37,-302.15)$.

The unit of velocity is $\mathrm{km} \mathrm{s}^{-1}$. In this model, the progenitor travels from north to south along the NW Stream with a perigalactic distance of $25.65 \mathrm{kpc}$. This demonstration is an updated version of their Case A simulation (see Figure 4 of Kirihara et al. 2017a).

Figure 16 shows an example of the $N$-body simulation of the NW Stream with our distance measurement, demonstrating that the simulation well represents the observation. To draw the distance distribution of the simulated NW Stream, we use the distribution of $\xi<0$ on the sky. Together with the results for the GCs, our observation strongly supports their simulation with the NW Stream as a background of M31. On the other hand, the latest PAndAS view (Richardson et al. 2011) suggests that the extension of the NW Stream is not clearly seen in their Figure 1 and may diminish or disappear when it passes the pericenter. This view is consistent with the case B simulation of Kirihara et al. (2017a). Therefore, searching for the southern extension of the NW Stream is important. Also, additional observation of the farther northern field is important because the simulated stream extends outside of the PAndAS field. Future fine-tuning of the simulation is planned to derive more accurate properties of the NW Stream (T. Kirihara et al. 2017 , in preparation). The key parameters from our survey are the following.

1. The luminosity distribution along the stream. According to Kirihara et al. (2017a), the $N$-body simulation can reproduce the observed features (i.e., the position of the NW Stream and the radial velocities for the five GCs) for several Myr to $1 \mathrm{Gyr}$, and it is difficult to determine the "current time" during the orbital motion of the NW Stream in the halo of M31. The promising information to solve this degeneracy is the luminosity distribution along the stream. The reason is that the simulated NW Stream has a higher surface brightness near the GC PAndAS-12 


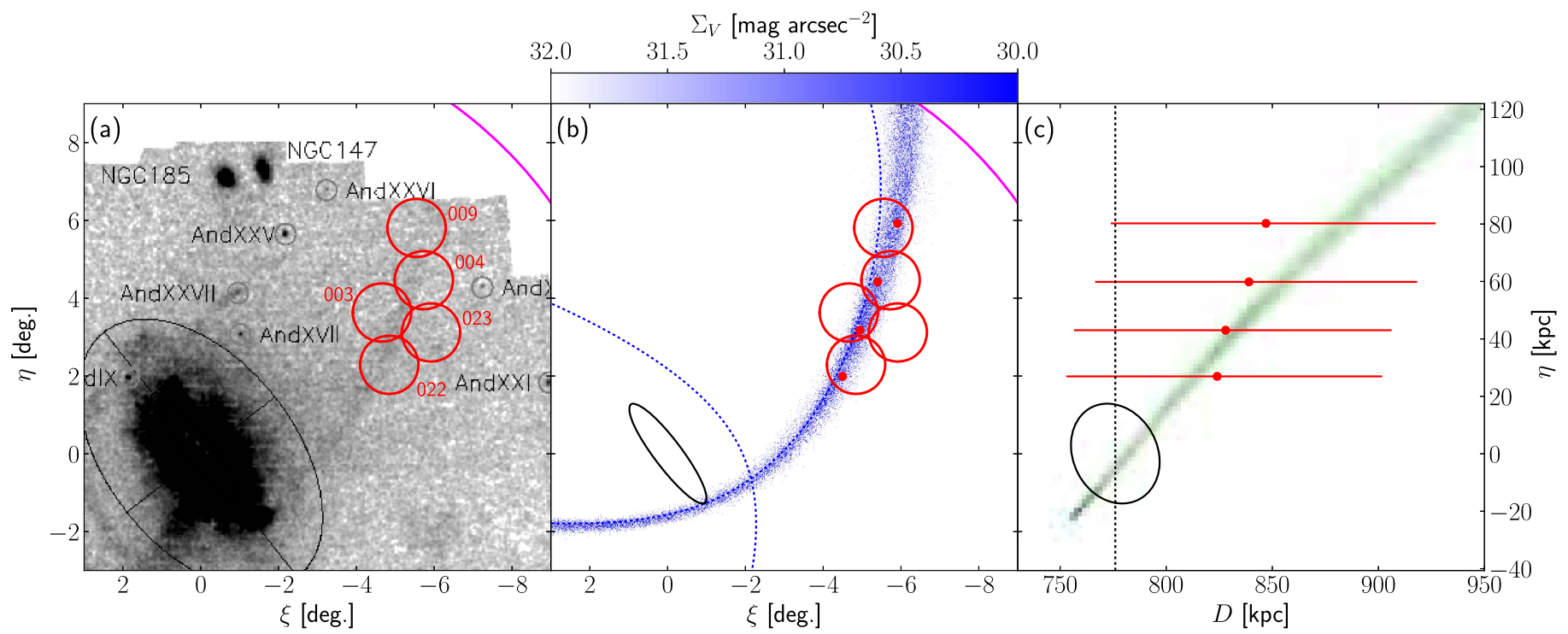

Figure 16. 3D schematic picture of the NW Stream. The optical image is shown in panel (a), which is the same as Figure 1. The particle distribution in panels (b) and (c) is a result of the $\mathrm{N}$-body simulation. M31 is represented as an ellipse with a major diameter of 190 arcmin assuming an inclination and position angle of $77^{\circ}$ and $37^{\circ}$ (Geehan et al. 2006), respectively. The HSC pointings are shown by the red circles in panel (b). The magenta curve represents $150 \mathrm{kpc}$ from the center of M31. The vertical black dashed line in panel (c) represents the distance to M31. The positions and distances of Stream 1-4 are shown by the red squares together with the error bars.

than the other parts. This trend is attributed to the selection of the "current time." Moreover, the position of the surviving central core of the progenitor gives a strong constraint because it depends on the properties and orbit of the progenitor (Kirihara et al. 2017a). As shown in Figures 7 and 13, our data provide sufficient information and would be helpful to reveal the nature of the NW Stream.

2. The width of the stream. As shown in Figure 15, the number of potential orbits of the NW Stream is still over several thousands, although it has been significantly reduced by making use of the line-of-sight distance to the stream. Therefore, other information to further constrain the orbits are required. By incorporating the width of the stream, the test-particle simulations will further constrain the allowable orbits. Figure 12 will be used for this purpose.

In relation to the above, it is emphasized that further observation along the NW Stream to both the north and south directions will be important. If the faint end of the stream is observed at the north, as suggested from the PAndAS map, it will be a very strong constraint. The observation in short exposure mode with the NB515 filter will be effective in answering this question.

\subsection{Diffuse Substructure}

The surface brightness of RC stars found at the diffuse substructure ranges from 34.8 to $36 \mathrm{mag} \mathrm{arcsec}^{-2}$ in the $g$ band (the median and brightest part are 35.5 and $34.8 \mathrm{mag} \mathrm{arcsec}^{-2}$, respectively). The total surface brightness of the diffuse substructure can be estimated from that of RC stars by assuming the an isochrone and initial mass function (IMF). The offset between the total surface brightness and the surface brightness of RC stars is calculated to be $1.69 \mathrm{mag}$ in the $g$ band for $Z=0.0014$ and the $\log \tau_{\text {age }}(\mathrm{Gyr})=10.00$ population with the Salpeter IMF. Therefore, the diffuse substructure would be observed to be 33.1-34.3 mag $\operatorname{arcsec}^{-2}$.
The surface brightness of $33 \mathrm{mag} \mathrm{arcsec}^{-2}$ is below the detection limit of the previous surveys based on the resolved stellar photometry such as PAndAS and Tanaka et al. (2010). Our result clearly shows that RC stars can be a powerful probe of the diffuse substructures down to $\sim 33 \mathrm{mag} \mathrm{arcsec}^{-2}$. Since the number of substructures is predicted to increase as the surface brightness becomes fainter (e.g., Bullock \& Johnston 2005), the actual appearance of the halo structure would be revealed in detail by tracing the RC stars. Only recently, the existence of an ultra-faint shell, having a common origin with the progenitor dwarf galaxy of the Giant Southern Stream, was predicted in the northwestern area of M31 (Kirihara et al. $2017 b$ ). The faint shell has similar properties to the diffuse substructure (surface brightness, distance, and metallicity), while there is a spatial offset of around $2^{\circ}$. It suggests that the diffuse substructures may be remnants of past galactic mergers. We therefore emphasize that photometric surveys down to the $\mathrm{RC}$ magnitude are very important to understand the assembly process of many subsystems that occurred in the outer part of the galaxy and to test the galaxy formation scenarios.

\section{Conclusions}

We have carried out a wide and deep imaging survey of the northwestern part of the halo in M31 using HSC on the Subaru Telescope. The survey covers a $9.2 \mathrm{deg}^{2}$ field consisting of five HSC pointings in the $g$ and $i$ bands as well as a narrowband filter, NB515. The reduced images in the $g, i$, and NB515 bands are deep enough with a mean $50 \%$ completeness limit of 26.31 , 25.69 , and $24.71 \mathrm{mag}$, respectively. The extinction-corrected CMD of our survey field exhibits characteristic features: a band of dwarf stars of the MW disk, a narrow sequence of a diffuse stellar stream in the MW halo, a broad sequence consisting of the RGB stars in the M31 halo, a diffuse but distinct concentration of BHB stars, and a significant RC feature.

The spatial distribution of RC stars shows a prominent stream feature, which is the known NW Stream. This substructure is also confirmed in the spatial distribution of 
RGB stars in the M31 halo, which are selected using NB515 photometry. The TRGB methodis applied to the NB515selected RGB stars. We obtain the distance modulus to the NW Stream based on the TRGB method to be $24.42 \pm 0.033$ (random) \pm 0.033 (systematic) mag, but the isochrone fitting to the CMD of Stream North suggests a larger distance modulus of $0.2-0.3$ mag. The distance estimates with the RC method show the distance moduli to be $24.63 \pm 0.191$ (random) \pm 0.057 (systematic) and $24.29 \pm 0.211$ (random) \pm 0.057 (systematic) mag for Stream North and Off-Stream South, respectively, indicating that the NW Stream is located behind the main body of M31, whereas the diffuse substructure is located in front of M31. We also estimate the line-of-sight distances along the NW Stream and find that the southern part of the NW Stream is $\sim 20$ $\mathrm{kpc}$ closer to us relative to the northern part. The number density distribution across the NW Stream represented by RC stars is found to be fitted by a Gaussian with FWHM of $\sim 25$ arcmin, but slightly skewed to the southwest side. The number density distribution of RC stars along the NW Stream shows the complicated structure, including the number of bumps and dips and a significant gap.

The stellar populations of GCs found in the NW Stream, PAndAS-10-13, are investigated based on the CMDs, and they are suggested to be similar to those composing the NW Stream. The 3D structure of the NW Stream revealed in this study is compared with the recent simulation carried out by Kirihara et al. (2017b) and gives a definite constraint on the allowable orbit of the NW Stream. The surface brightness of the diffuse substructure found in the southern part of the survey field is estimated to be $\sim 33 \mathrm{mag} \operatorname{arcsec}^{-2}$ in the $g$ band, which is below the detection limit of the previous surveys of the M31 halo. We have found that the RC stars are a powerful probe to assess diffuse substructures, which are expected to be numerous and to reveal the real appearance of the M31 halo.

This work is supported in part by the JSPS Grant-in-Aid for Scientific Research (No. JP25287062, JP15K05037, JP25800098, JP25400222, and JP17H01101), MEXT Grant-in-Aid for Scientific Research on Innovative Areas (No. JP15H05892, JP16H01090 for K.H., JP16H01086 for M.C.), and Grant-inAid for JSPS Fellows (TK 26.348). P.G. was supported by NSF grant AST-1412648. M.G.L. and I.S.J. were supported by the National Research Foundation (NRF) grant funded by the Korea Government (NRF-2017R1A2B4004632).

We would like to thank all the staff of Subaru Telescope, in particular Drs. Fumiaki Nakata, Tsuyoshi Terai, Shintaro Koshida, Francois Finet, and Akito Tajitsu for their excellent support during the observation, and all the staff of the HSC software developing group, in particular Dr. Hisanori Furusawa and Dr. Naoki Yasuda, for their advice during the processing of our M31 data. We would like to express our appreciation to Dr. Satoshi Kawanomoto for his effort in developing the NB515 filter. We are grateful to Dr. Paul Price for his kind support in calibrating our data to the PS1 system. We appreciate the many valuable comments and suggestions from the referee, which have improved this paper significantly.

The numerical computations were carried out on the HAPACS System in the Center for Computational Sciences, University of Tsukuba, Japan.

The HSC collaboration includes the astronomical communities of Japan and Taiwan, and Princeton University. The HSC instrumentation and software were developed by the National
Astronomical Observatory of Japan (NAOJ), the Kavli Institute for the Physics and Mathematics of the Universe (Kavli IPMU), the University of Tokyo, the High Energy Accelerator Research Organization (KEK), the Academia Sinica Institute for Astronomy and Astrophysics in Taiwan (ASIAA), and Princeton University. Funding was contributed by the FIRST program from the Japanese Cabinet Office, the Ministry of Education, Culture, Sports, Science and Technology (MEXT), the Japan Society for the Promotion of Science (JSPS), Japan Science and Technology Agency (JST), the Toray Science Foundation, NAOJ, Kavli IPMU, KEK, ASIAA, and Princeton University. This paper makes use of software developed for the Large Synoptic Survey Telescope. We thank the LSST Project for making their code freely available. The Pan-STARRS1 (PS1) Surveys have been made possible through contributions of the Institute for Astronomy, the University of Hawaii, the Pan-STARRS Project Office, the Max-Planck Society and its participating institutes, the Max Planck Institute for Astronomy and the Max Planck Institute for Extraterrestrial Physics, The Johns Hopkins University, Durham University, the University of Edinburgh, Queen's University Belfast, the HarvardSmithsonian Center for Astrophysics, the Las Cumbres Observatory Global Telescope Network Incorporated, the National Central University of Taiwan, the Space Telescope Science Institute, the National Aeronautics and Space Administration under grant No. NNX08AR22G issued through the Planetary Science Division of the NASA Science Mission Directorate, the National Science Foundation under grant No. AST-1238877, the University of Maryland, and Eotvos Lorand University (ELTE).

\section{Appendix \\ Transformation between the HSC and Other Standard Photometric Systems}

Photometric transformations are required to compare our results with previous studies, which were carried out in different filter systems. In this section, the color conversion formulae relevant to our study are summarized. The method to obtain the formulae is similar to those used in previous studies, such as Yagi et al. (2010) and Fukugita et al. (1995). We calculate the colors of stars using the Bruzual-Persson-GunnStryker (BPGS) Atlas by convolving the transmission curve of a filter system (Johnson-Cousins system for Bessell 1990 and SDSS system for Doi et al. 2010) to the stellar spectra. The transmission curves for HSC can be obtained from the Subaru Telescope HSC Web site. ${ }^{23}$

Figure 17 shows our calculation. The top panel of each of the figure sections (a)-(d) shows the calculated colors $\left(V-I_{C}\right.$, $\left.i_{\mathrm{HSC}}-I_{C}, B-V, g_{\mathrm{HSC}}-V\right)$ for BPGS stars plotted against $(g-i)_{\mathrm{HSC}}$. The best-fit functions, linear expression and quadratic expression, are plotted as the green and cyan dashed lines, respectively. The fitting to the quadratic expression is performed for $-0.5<(g-i)_{\mathrm{HSC}}<2.0$, but that to the linear expression is for a more limited color range $(1.3<$ $\left.(g-i)_{\mathrm{HSC}}<1.7\right)$, which is relevant to this study. In the middle and bottom panels, the residuals from the quadratic and linear fits, respectively, are plotted against $(g-i)_{\mathrm{HSC}}$.

The fitting for $i_{\mathrm{HSC}}-I_{C}$ is performed very well with root mean square (rms) residuals of 0.008-0.009, and those for $g_{\mathrm{HSC}}-V$ and $V-I_{C}$ are fair with an rms of $\sim 0.019$. That for

\footnotetext{
${ }^{23}$ http://www.naoj.org/Observing/Instruments/HSC/index.html
} 


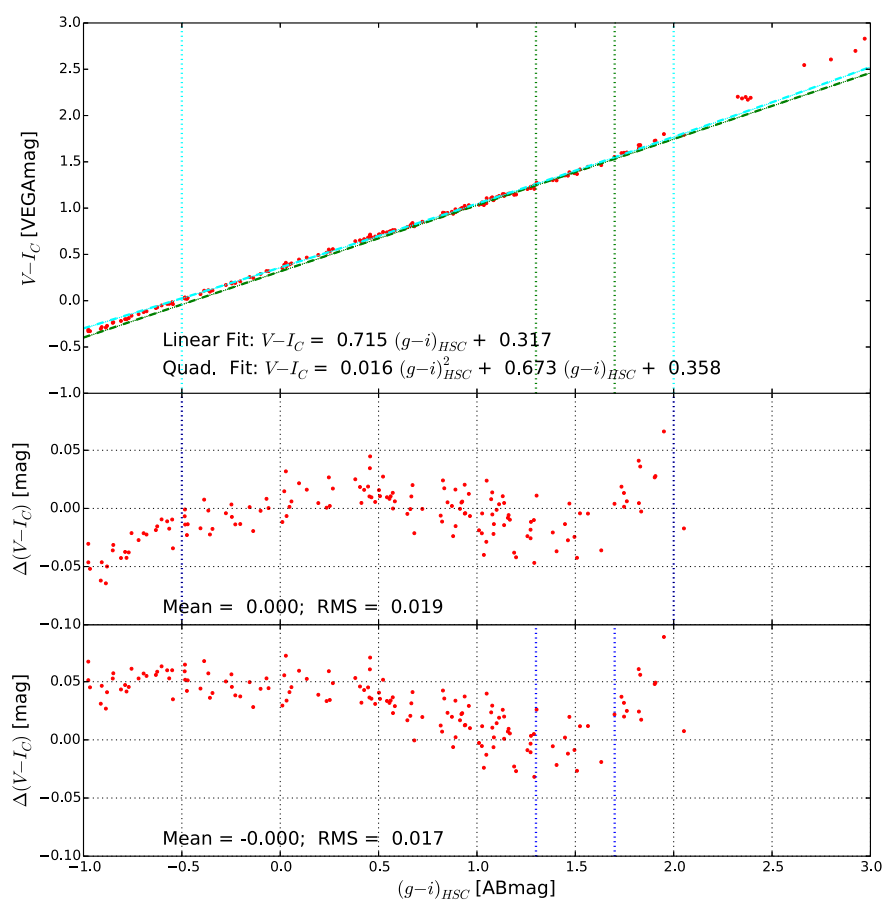

(a)

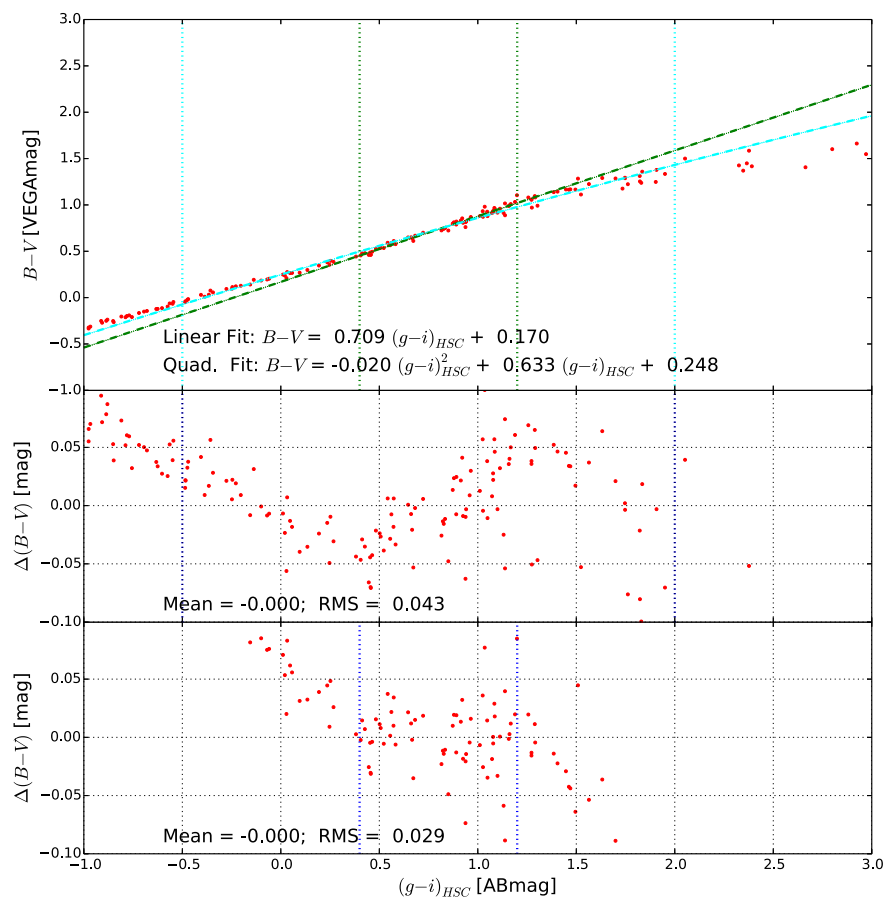

(c)

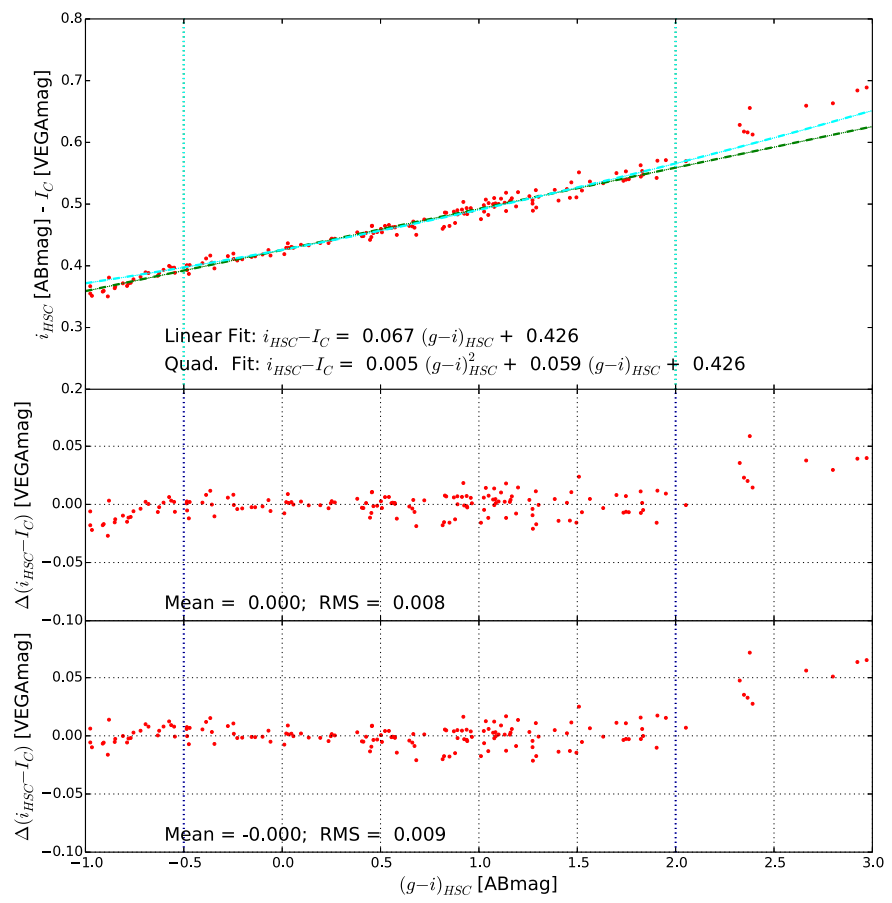

(b)

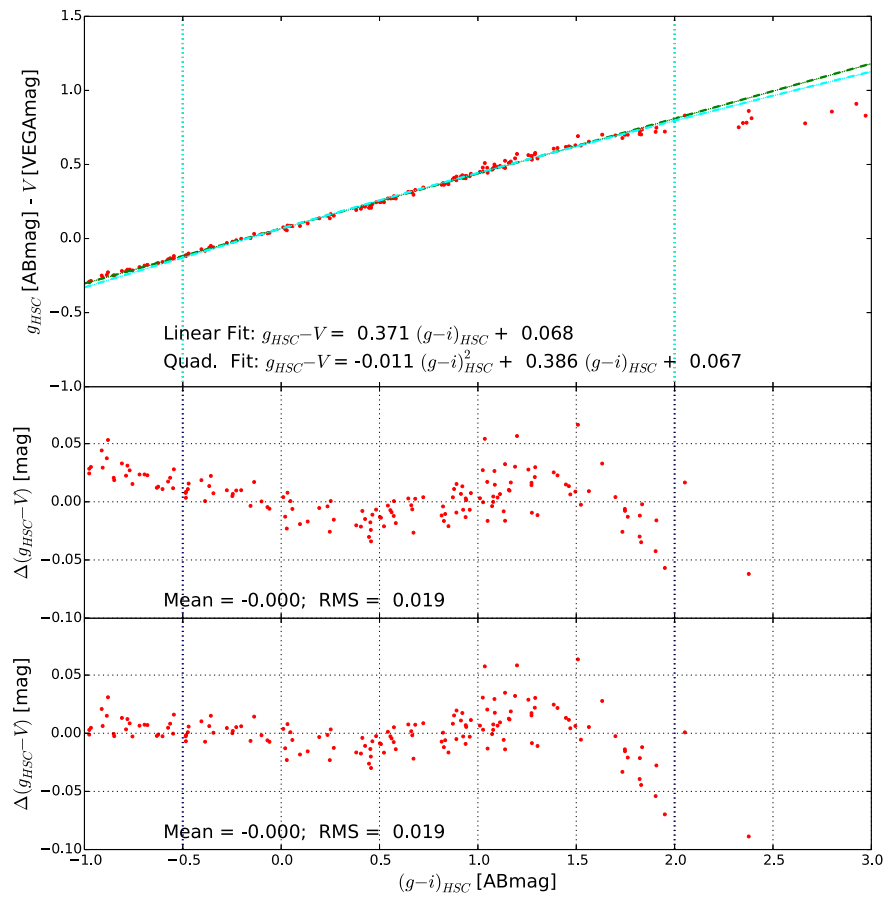

(d)

Figure 17. Color conversion diagrams between (a) $(g-i)_{\mathrm{HSC}}$ and $V-I_{C}$, (b) $(g-i)_{\mathrm{HSC}}$ and $i_{\mathrm{HSC}}-I_{C},(\mathrm{c})(g-i)_{\mathrm{HSC}}$ and $B-V$, and (d) $(g-i)_{\mathrm{HSC}}$ and $g_{\mathrm{HSC}}-V$.

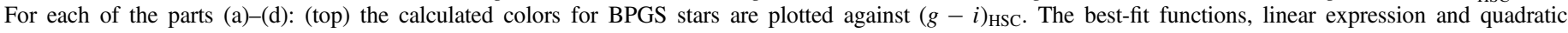
expression, are plotted as green and cyan dashed lines and the color range used for the fitting is plotted as vertical dotted lines with the same color, respectively. (Middle) The residuals of the calculated color from the quadratic fit. The blue vertical dotted lines indicate the color range used for the fitting. (Bottom) Same as for the middle panel, but for the linear fit.

$B-V$ is the worst, but the rms is reasonable if the color range is limited to $0.4<(g-i)_{\mathrm{HSC}}<1.2$, which is sufficient for this study. In this study, we use

$$
\begin{aligned}
& V-I_{C}=0.715(g-i)_{\mathrm{HSC}}+0.317 \\
& \quad \text { for } 1.3<(g-i)_{\mathrm{HSC}}<1.7
\end{aligned}
$$

$$
\begin{gathered}
i_{\mathrm{HSC}}-I_{C}=0.067(g-i)_{\mathrm{HSC}}+0.426, \\
B-V=0.709(g-i)_{\mathrm{HSC}}+0.170 \\
\text { for } 0.4<(g-i)_{\mathrm{HSC}}<1.2, \\
g_{\mathrm{HSC}}-V=0.371(g-i)_{\mathrm{HSC}}+0.068
\end{gathered}
$$

for the analysis. 


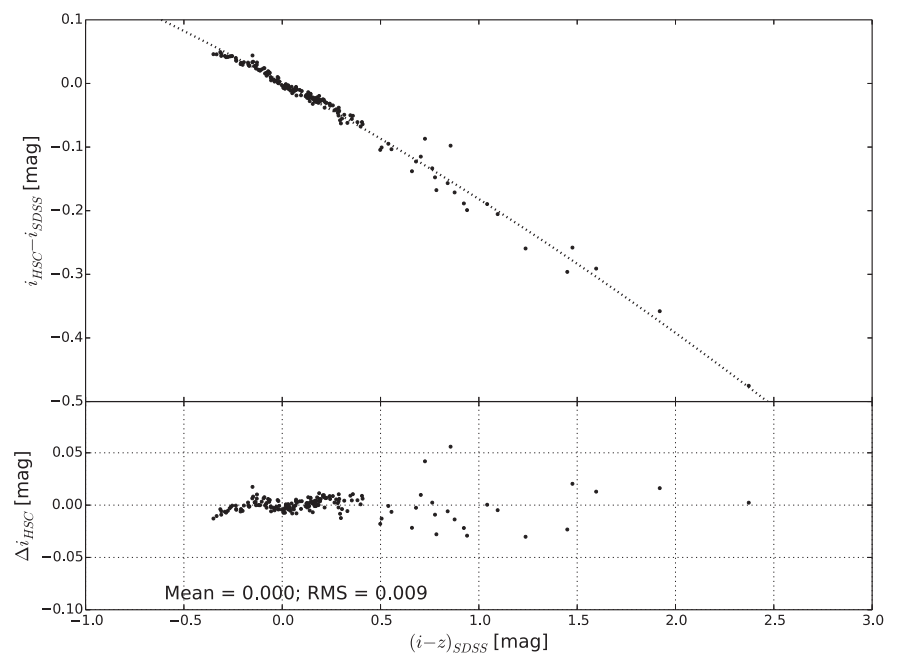

(a) $(i-z)_{S D S S}$ versus $i_{H S C}$

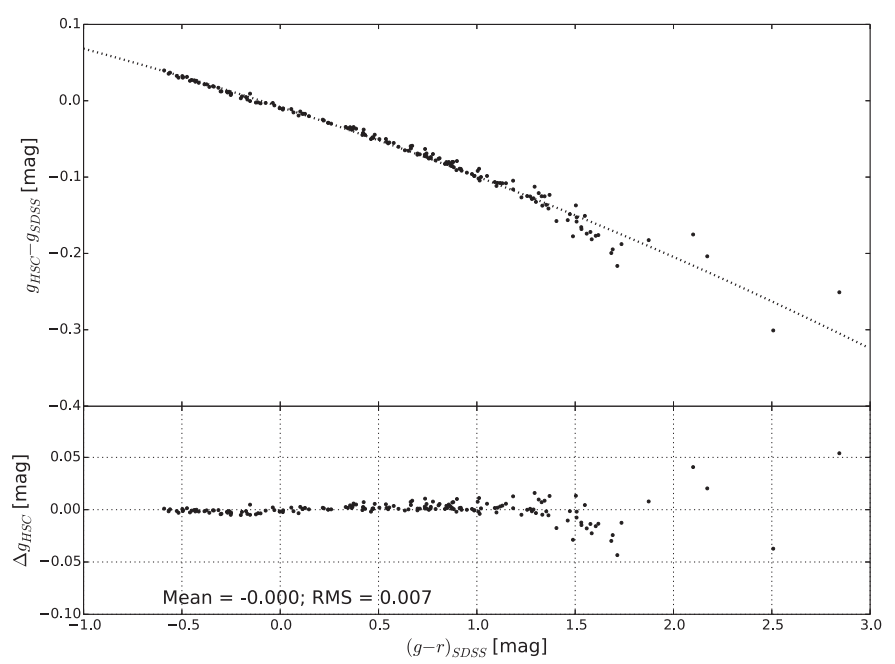

(c) $(g-r)_{S D S S}$ versus $g_{H S C}$

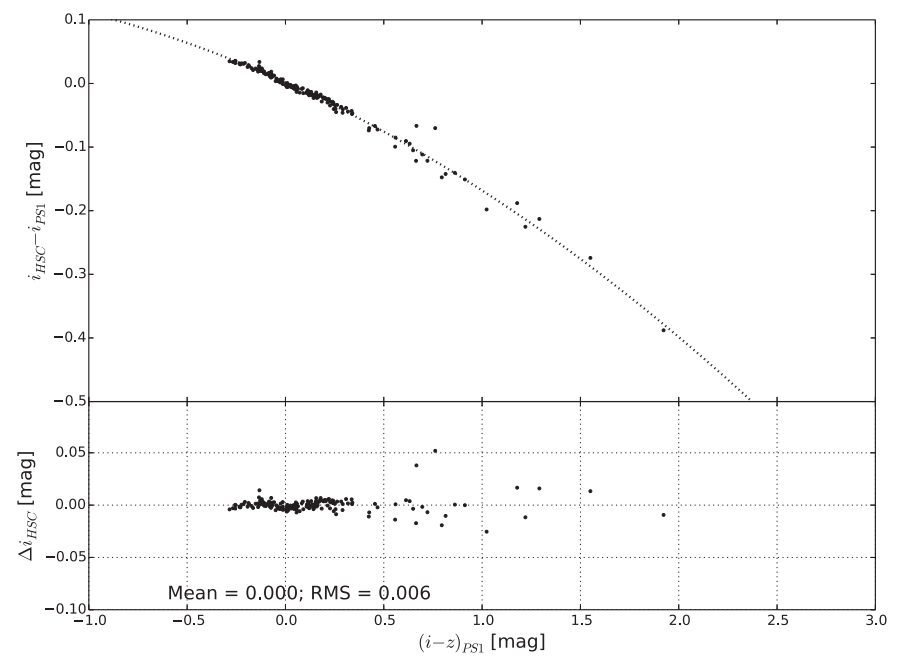

(b) $(i-z)_{P S 1}$ versus $i_{H S C}$

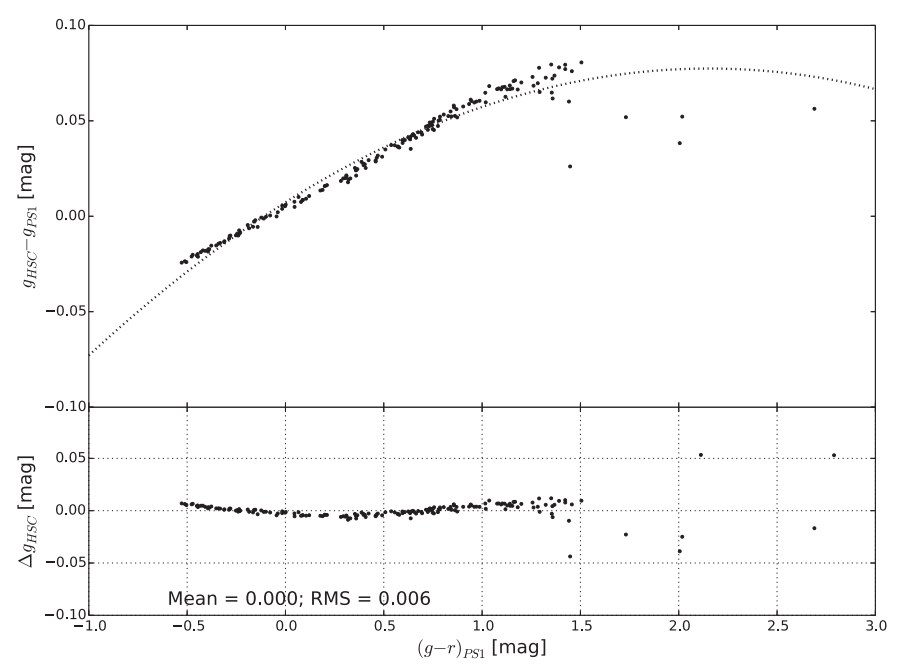

(d) $(g-r)_{P S 1}$ versus $g_{H S C}$

Figure 18. Color conversion diagrams between (a) $(i-z)_{\mathrm{SDSS}}$ and $i_{\mathrm{HSC}}$, (b) $(i-z)_{\mathrm{PS} 1}$ and $i_{\mathrm{HSC}}$, (c) $(g-r)_{\mathrm{SDSS}}$ and $g_{\mathrm{HSC}}$, and (d) $(g-r)_{\mathrm{PS} 1}$ and $g_{\mathrm{HSC}}$. For each of the parts (a)-(d): (top) the magnitude difference of the $g$ and $i$ bands (i.e., color terms) between HSC and either the SDSS or PS1 systems calculated for BPGS stars as a function of color. The dotted line shows the quadratic expression of the data, which is one of Equations (15)-(18). (Bottom) The magnitude difference between the HSC magnitude and that calculated with one of Equations (15)-(18). The rms value is calculated for stars with moderate color (i.e., $(g-r)$ SDSS,PS $1<2.0$ or $\left.(i-z)_{\text {SDSS,PS1 }}<1.0\right)$ and listed in the figure.

We also use the color conversion formulae from the SDSS system to the HSC system,

$$
\begin{aligned}
i_{\mathrm{HSC}}= & i_{\mathrm{SDSS}}+0.00130204-0.16922042\left(i_{\mathrm{SDSS}}-z_{\mathrm{SDSS}}\right) \\
& -0.01374245\left(i_{\mathrm{SDSS}}-z_{\mathrm{SDSS}}\right)^{2},
\end{aligned}
$$

$$
\begin{aligned}
g_{\mathrm{HSC}}= & g_{\mathrm{SDSS}}-0.00816446-0.08366937 \\
& \times\left(g_{\mathrm{SDSS}}-r_{\mathrm{SDSS}}\right)-0.00726883\left(g_{\mathrm{SDSS}}-r_{\mathrm{SDSS}}\right)^{2},
\end{aligned}
$$

which are adopted in hscPipe when converting the isochrone described in the SDSS system.

During the image reduction, the HSC images are calibrated against PS1 using the following color conversion formulae,

$$
\begin{aligned}
i_{\mathrm{HSC}}= & i_{\mathrm{PS} 1}+0.00166891-0.13944659\left(i_{\mathrm{PS} 1}-z_{\mathrm{PS} 1}\right) \\
& -0.03034094\left(i_{\mathrm{PS} 1}-z_{\mathrm{PS} 1}\right)^{2},
\end{aligned}
$$

$$
\begin{aligned}
g_{\mathrm{HSC}}= & g_{\mathrm{PS} 1}+0.00730066+0.06508481\left(g_{\mathrm{PS} 1}-r_{\mathrm{PS} 1}\right) \\
& -0.0151057\left(g_{\mathrm{PS} 1}-r_{\mathrm{PS} 1}\right)^{2} .
\end{aligned}
$$

The top panels of the four sections (a)-(d) of Figure 18 show the magnitude difference of the $g$ and $i$ bands (i.e., color terms) between HSC and either the SDSS or PS1 systems calculated for BPGS stars as a function of color. These panels indicate that the magnitude difference can be represented as quadratic expressions of color for wide color ranges. The bottom panels of the four sections (a)-(d) of Figure 18 show the difference between the HSC magnitude and that calculated using Equations (15)-(18). The rms is calculated for stars with moderate color (i.e., $(g-r)_{\mathrm{SDSS}, \mathrm{PS} 1}<2.0$ or $\left.(i-z)_{\mathrm{SDSS}, \mathrm{PS} 1}<1.0\right)$ and found to be less than $0.01 \mathrm{mag}$. 


\section{ORCID iDs}

Masashi Chiba (1) https://orcid.org/0000-0002-9053-860X Masayuki Tanaka (i) https://orcid.org/0000-0002-5011-5178 Robert H. Lupton (1) https://orcid.org/0000-0003-1666-0962 Puragra Guhathakurta (i) https://orcid.org/0000-0001-8867-4234 Karoline Gilbert (1) https://orcid.org/0000-0003-0394-8377 Evan Kirby (1) https://orcid.org/0000-0001-6196-5162 Sanjib Sharma (1) https://orcid.org/0000-0002-0920-809X Kohei Hayashi 누 https://orcid.org/0000-0002-8758-8139

\section{References}

Alvez, D. R., \& Sarajedini, A. 1999, ApJ, 511, 225

Belokurov, V., Zucker, D. B., Evans, N. W., et al. 2006, ApJL, 642, L137 Bessell, M. S. 1990, PASP, 102, 1181

Bilir, S., Ak, T., Ak, S., Yontan, T., \& Bostanci, Z. F. 2013, NewA, 23, 88 Bosch, J., Armstrong, R., Bickerton, S., et al. 2017, PASJ, in press Bressan, A., Marigo, P., Girardi, L., et al. 2012, MNRAS, 427, 127 Bullock, J. S., \& Johnston, K. V. 2005, ApJ, 635, 931

Carlberg, R. G. 2012, ApJ, 748, 20

Carlberg, R. G., Richer, H. R., McConnachie, A. W., et al. 2011, ApJ, 731, 124

Castelli, F., \& Kurucz, R. L. 2004, arXiv:astro-ph/0405087

Chabrier, G. 2001, ApJ, 554, 1274

Conn, A. R., Lewis, G. F., Ibata, R. A., et al. 2011, ApJ, 740, 69

Cooper, A. P., Cole, S., Frenk, C. S., et al. 2010, MNRAS, 406, 744

Doi, M., Tanaka, M., Fukugita, M., et al. 2010, AJ, 139, 1628

Erkal, D., Belokurov, V., Bovy, J., \& Sanders, J. L. 2016, MNRAS, 463, 102 Fardal, M. A., Guhathakurta, P., Babul, A., \& McConnachie, A. W. 2007, MNRAS, 380, 15

Feltzing, S., \& Chiba, M. 2013, NewAR, 57, 80

Font, A. S., McCarthy, I. G., Crain, R. A., et al. 2011, MNRAS, 416, 2802 Fukugita, M., Shimasaku, K., \& Ichikawa, T. 1995, PASP, 107, 945 Furusawa, H., Koike, M., Takata, T., et al. 2017, PASJ, in press

Geehan, J. J., Fardal, M. A., Babul, A., \& Guhathakurta, P. 2006, MNRAS, 366, 996

Gilbert, K. M., Guhathakurta, P., Beaton, R. L., et al. 2012, ApJ, 760, 76

Gilbert, K. M., Guhathakurta, P., Kalirai, J. S., et al. 2006, ApJ, 652, 1188

Gilbert, K. M., Kalirai, J. S., Guhathakurta, P., et al. 2014, ApJ, 796, 76

Gilmore, G., Wilkinson, M. I., Wyse, R. F. G., et al. 2007, ApJ, 663, 948

Guhathakurta, P., Ostheimer, J. C., Gilbert, K. M., et al. 2005, arXiv:astro-ph/ 0502366

Gunn, J. E., \& Stryker, L. L. 1983, ApJS, 52, 121

Harris, W. E. 1996, AJ, 112, 1487

Hernquist, L. 1990, ApJ, 356, 359

Huxor, A. P., Mackey, A. D., Ferguson, A. M. N., et al. 2014, MNRAS, 442, 2165

Ibata, R. A., Lewis, G. F., Conn, A. R., et al. 2013, Natur, 493, 62

Ibata, R. A., Lewis, G. F., McConnachie, A. W., et al. 2014, ApJ, 780, 128

Ivezic, Z., Axelrod, T., Brandt, W. N., et al. 2008, AJ, 176, 1

Jang, I. S., \& Lee, M. G. 2017, ApJ, 835, 28

Jester, S., Schneider, D. P., Richards, G. T., et al. 2005, AJ, 130, 873

Juric, M., Ivezic, Z., Brooks, A., et al. 2008, ApJ, 673, 864
Juric, M., Kantor, J., Lim, K.-T., et al. 2015, arXiv:1512.07914

Kalirai, J. S., Gilbert, K. M., Guhathakurta, P., et al. 2006, ApJ, 648, 389

Kawanomoto, S., Uraguchi, F., Komiyama, Y., et al. 2017, PASJ, in press

Kirihara, T., Miki, Y., \& Mori, M. 2017a, MNRAS, 469, 3390

Kirihara, T., Miki, Y., Mori, M., Kawaguchi, T., \& Rich, R. M. 2017b, MNRAS, 464, 3509

Komiyama, Y., Obuchi, Y., Nakaya, H., et al. 2017, PASJ, in press

Koposov, S., Belokurov, V., Evans, N. W., et al. 2008, ApJ, 686, 279

Koposov, S. E., Belokurov, V., Torrealba, G., Evans, N. W., et al. 2015, ApJ, 805,130

Kroupa, P. 2002, Sci, 295, 82

Küpper, A. H. W., Lane, R. R., \& Heggie, D. C. 2012, MNRAS, 420, 2700

Macciò, A. V., \& Fontanot, F. 2010, MNRAS, 404, L16

Magnier, E. A., Schlafly, E., Finkbeiner, D., et al. 2013, ApJS, 205, 20

Majewski, S. R., Ostheimer, J. C., Kunkel, W. E., \& Patterson, R. J. 2000, AJ, 120,2550

Martin, N. F., de Jong, J. T. A., Rix, H.-W., et al. 2008, ApJ, 684, 1075

Martin, N. F., Ibata, R. A., Rich, R. M., et al. 2014, ApJ, 787, 19

McConnachie, A. W. 2012, AJ, 144, 4

McConnachie, A. W., Irwin, M. J., Ibata, R. A., et al. 2009, Natur, 461, 66 Miki, T., \& Umemura, M. 2017, arXiv:1712.08760

Miyazaki, S., Komiyama, Y., Kawanomoto, S., et al. 2017, PASJ, in press

Miyazaki, S., Komiyama, Y., Nakaya, H., et al. 2012, Proc. SPIE, 8446, $84460 \mathrm{Z}$

Morrison, H. L., Mateo, M., Olszewski, E. W., Harding, P., Dohm-Palmer, R. C., Freeman, K. C., Norris, J. E., \& Morita, M. 2000, AJ, 119, 2254

Navarro, J. F., Frenk, C. S., \& White, S. D. M. 1998, ApJ, 462, 563

Pickles, A. J. 1998, PASP, 110, 863

Richardson, J. C., Irwin, M. J., McConnachie, A. W., et al. 2011, ApJ, 732, 76

Rizzi, L., Tully, R. B., Makarov, D., et al. 2007, ApJ, 661, 815

Sakai, S., Madore, B. F., \& Freedman, W. L. 1996, ApJ, 461, 713

Sakamoto, T., \& Hasegawa, T. 2006, ApJL, 653, L29

Salpeter, E. E. 1955, ApJ, 121, 161

Sawala, T., Frenk, C. S., Fattahi, A., et al. 2016, MNRAS, 457, 1931

Schlafly, E. F., \& Finkbeiner, D. P. 2011, ApJ, 737, 103

Schlafly, E. F., Finkbeiner, D. P., Jurić, M., et al. 2012, ApJ, 756, 158

Schlegel, D. J., Finkbeiner, D. P., \& Davis, M. 1998, ApJ, 500, 525

Simon, J. D., \& Geha, M. 2007, ApJ, 670, 313

Stetson, P. B. 1987, PASP, 99, 191

Stetson, P. B. 1994, PASP, 106, 250

Tanaka, M., Chiba, M., Komiyama, Y., et al. 2010, ApJ, 708, 1168

Tanaka, M., Chiba, M., \& Komiyama, Y. 2017, ApJ, 842, 127

Tollerud, E. J., Bullock, J. S., Strigari, L. E., \& Willman, B. 2008, ApJ, 688, 277

Tollerud, E. J., Geha, M. C., Grcevich, J., Putman, M. E., Weisz, D. R., \& Dolphine, A. E. 2016, ApJ, 827, 89

Tonry, J. L., Stubbs, C. W., Lykke, K. R., et al. 2012, ApJ, 750, 99

Veljanoski, J., Mackey, A. D., Ferguson, A. M. N., et al. 2014, MNRAS, 442, 2929

Walsh, S. M., Willman, B., \& Jerjen, H. 2009, AJ, 137, 45

Willman, B., Blanton, M. R., West, A. A., et al. 2005, AJ, 129, 2692

Yagi, M., Yoshida, M., Komiyama, Y., et al. 2010, AJ, 140, 1814

York, D. G., Adelman, J., Anderson, J. E., Jr., et al. 2000, AJ, 120, 1579 